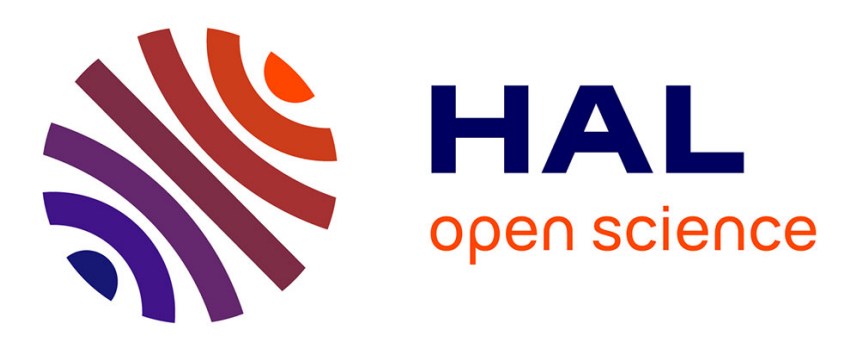

\title{
Towards a global control strategy for Induction motor Speed regulation: flux optimization and power factor correction
}

\author{
A. El Fadili, Fouad Giri, Abdelmounime El Magri, R. Lajouad, Fatima Zara \\ Chaoui
}

\section{To cite this version:}

A. El Fadili, Fouad Giri, Abdelmounime El Magri, R. Lajouad, Fatima Zara Chaoui. Towards a global control strategy for Induction motor Speed regulation: flux optimization and power factor correction. International Journal of Electrical Power \& Energy Systems, 2012, 43 (1), pp.Pages 230244. 10.1016/j.ijepes.2012.05.022 . hal-01061750

\section{HAL Id: hal-01061750 \\ https://hal.science/hal-01061750}

Submitted on 12 Sep 2014

HAL is a multi-disciplinary open access archive for the deposit and dissemination of scientific research documents, whether they are published or not. The documents may come from teaching and research institutions in France or abroad, or from public or private research centers.
L'archive ouverte pluridisciplinaire HAL, est destinée au dépôt et à la diffusion de documents scientifiques de niveau recherche, publiés ou non, émanant des établissements d'enseignement et de recherche français ou étrangers, des laboratoires publics ou privés. 


\title{
Towards a global control strategy for induction motor: Speed regulation, flux optimization and power factor correction
}

\author{
A. El Fadili *, F. Giri, A. El Magri, R. Lajouad, F.Z. Chaoui \\ GREYC Lab., University of Caen, Caen, France
}

Keywords:

Induction machine

Magnetic circuit nonlinearity

AC/DC/AC converters

Speed regulation

Power factor correction

Backstepping technique

\begin{abstract}
A B S T R A C T
A great deal of interest has been paid to induction machine control over the last years. However, most previous works have focused on the speed/flux/torque regulation supposing the machine magnetic circuit to be linear and ignoring the machine power conversion equipments. The point is that speed regulation cannot be ensured in optimal efficiency conditions, for a wide range of speed-set-point and load torque, unless the magnetic circuit nonlinearity is explicitly accounted for in the motor model. On the other hand, the negligence of the power conversion equipments makes it impossible to deal properly with the harmonic pollution issue due to 'motor - power supply grid' interaction. This paper presents a theoretical framework for a global control strategy of the induction machine and related power equipments. The proposed strategy involves a multi-loop nonlinear adaptive controller designed to meet the three main control objectives, i.e. tight speed regulation for a wide range speed-reference variation, flux optimization for energy consumption and power factor correction (PFC). Tools from the averaging theory are resorted to formally describe the control performances.
\end{abstract}

\section{Introduction}

Induction motors are featured by their interesting power/mass ratio, relatively low cost and simple maintenance (as they include no mechanical commutator). It is widely agreed that these machines have promising perspectives in the industrial actuator field. This has motivated an intensive research activity on induction machine control especially over the last fifteen years. The complexity of this problem is threefold:

- The multivariable and nonlinear nature of the machine dynamics.

- The multiform interaction with its environment: supply grid, power converters, varying load, etc.

- The multiplicity of control objectives: speed regulation, energy consumption optimization, power factor correction, fault detection and diagnostic, etc.

Most previous works have focused only on speed/flux regulation (with constant flux reference) following several control strategies ranging from simple techniques, e.g. field-oriented control $[11,14]$, to more sophisticated nonlinear approaches, e.g. feedback linearization [1], direct torque control $[21,4]$ or sliding mode

\footnotetext{
* Corresponding author.

E-mail address: elfadili_abderrahim@yahoo.fr (A. El Fadili).
}

control [19]. A common point of these works is that the control design relies on a relatively simple machine model, next called standard model, assuming a linear representation of the magnetic circuit (which of course is not true in real machines). Accordingly, most previous control solutions involved flux regulation around constant values (Fig. 1). Specifically, the constant flux reference is equal to its nominal value generally located at the elbow of the machine magnetic characteristic [12,2]. Then, energetic efficiency is actually maximal provided that the machine operates all time in the neighborhood of its nominal point. But, this is not the case in most practical applications because the machine load is generally varying [8]. Indeed, in presence of small loads, the operation point is below the nominal value causing useless energy stored in stator inductances which reduces the machine efficiency. In the case of overloaded machine, this operates in the saturation zone of its magnetic characteristic but, then, the standard model is no longer valid and, consequently, the control performances are no longer guaranteed. To overcome the above shortcomings, it is necessary in speed control to let the flux reference be dependent on both the speed reference and torque-load, i.e. the flux reference must be state-dependent. Examples of speed/flux controllers involving state-dependent flux reference (Fig. 2) have been developed in [15]. The proposed controllers include optimal flux generators the design of which relies on a machine model that takes into account the nonlinearity of the magnetic characteristic.

However, even in the preceding works the control problem has been relatively simplified because the motor is viewed there as a 


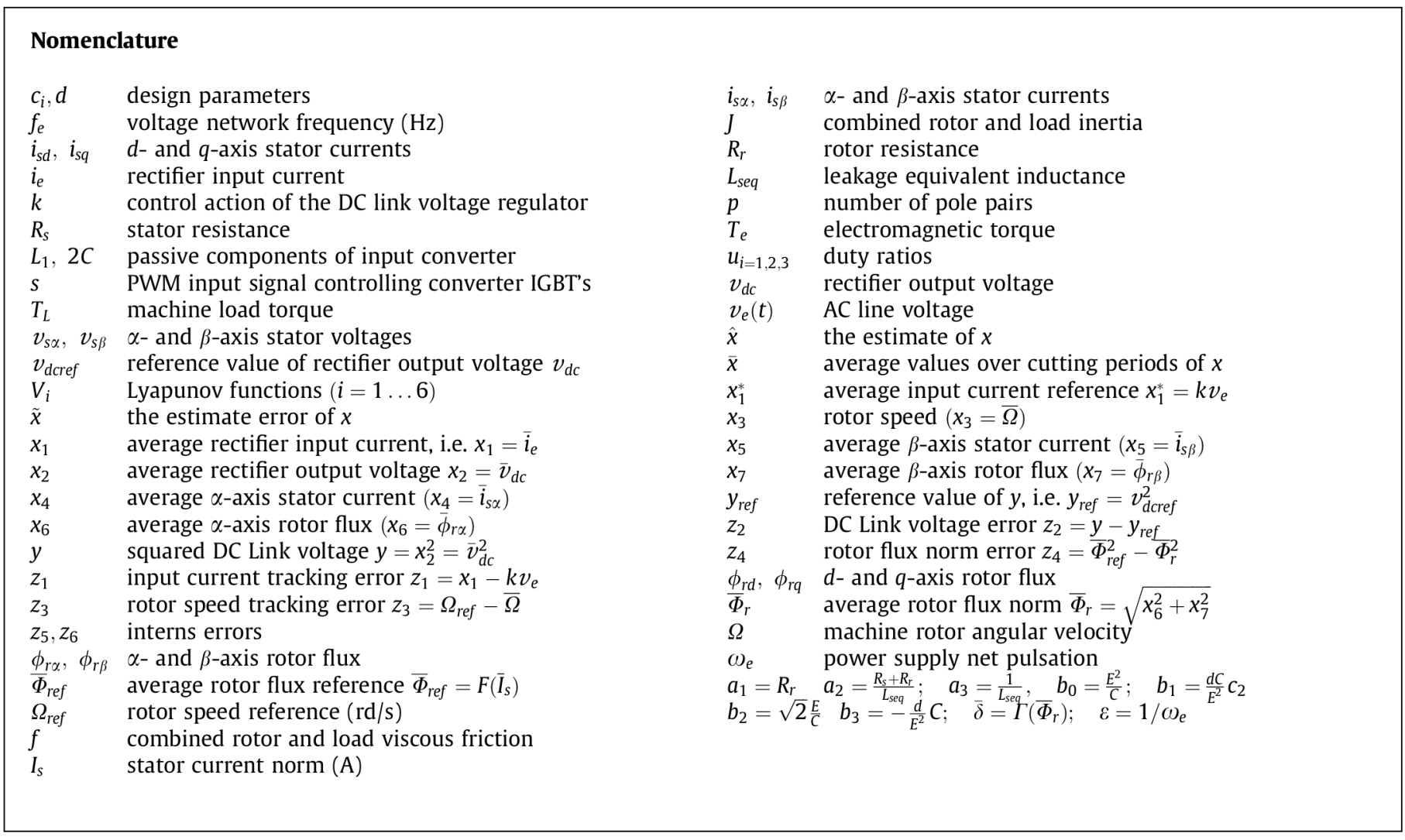

separated system directly controlled by acting on the stator voltages. As a matter of fact, in practical applications, the motor is physically controlled through a (three-phase) DC/AC PWM switch inverter. Furthermore, the inverter is connected to a power supply net through a AC/DC PWM rectifier (Fig. 3). Ignoring the latter amounts to suppose that the DC-link voltage (i.e. the AC/DC rectifier output voltage) is perfectly regulated. The point is that perfect regulation of such voltage cannot be ensured ignoring the rectifier load which is nothing other than the set 'DC/AC inverter-Motor'. Moreover, the Rectifier-Inverter-Motor association strongly interacts with the AC power supply net (Fig. 3). Accordingly, the power flow is in fact bidirectional: the circulation sense depends on the load variation. Then, undesirable current harmonics are likely to be generated in the $\mathrm{AC}$ line, due to the strongly nonlinear nature of the association 'converter-inverter-motor'. This harmonic pollution has several damaging effects on the quality of power distribution along the $\mathrm{AC}$ line, e.g. electromagnetic compatibility issues, voltage distortion, reactive power increase, larger power losses, increased voltage drops, etc. In this respect, standards such

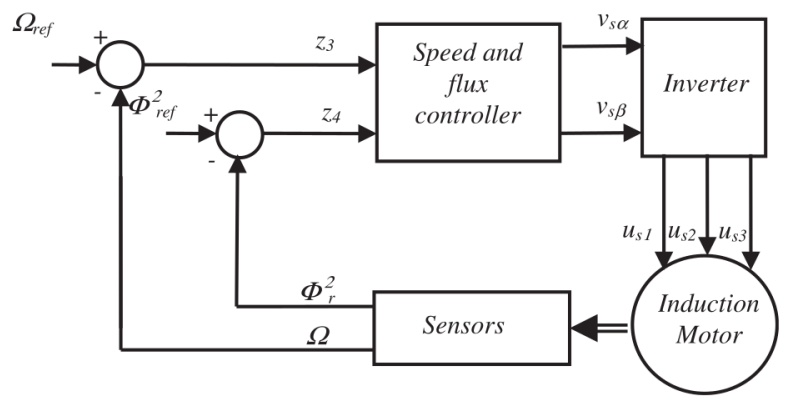

Fig. 1. Control strategy involving constant flux reference (the controller is obtained from the standard model).

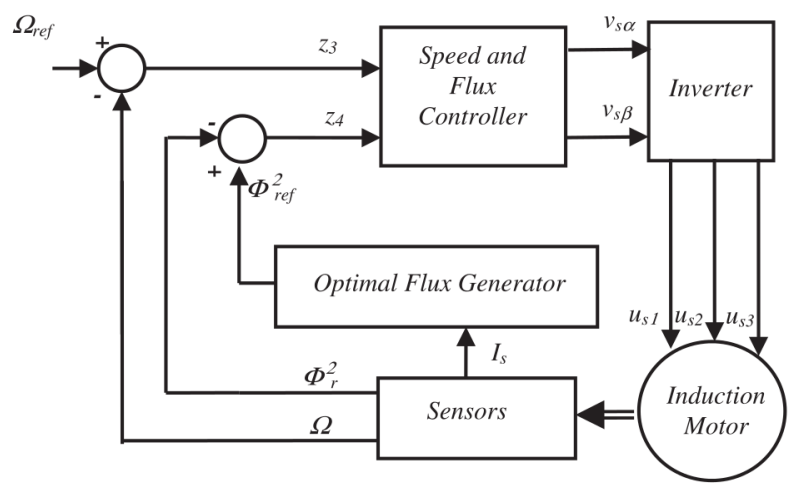

Fig. 2. Control strategy involving state-dependent optimal flux (SDOF) reference.

as IEEE519-1992 and IEC 61000-3-2/IEC 6100-3-4 indicate the current harmonic limits to be complied with, in terms of power factor correction $[9,18]$. Of course, the power factor can be improved using additional protection equipments (transformers, condensers, etc.) and/or over-dimensioning the converter and net elements. However, this solution is costly and may not be sufficient.

An attempt to simultaneously deal with speed/flux control and PFC requirement has been done [16]. But, the flux optimization requirement was not coped with there.

In the light of the above remarks, it becomes clear that a convenient control strategy is one that consists in dealing with the control problem for the whole association 'rectifier-inverter-motor', seeking simultaneous achievement of all relevant control objectives, i.e. tight speed regulation for wide set-point variation range, flux optimization despite large load change, satisfactory power factor correction (PFC), fault detection and diagnostic, etc. Such a global control strategy has still to be developed (Fig. 4). This study 


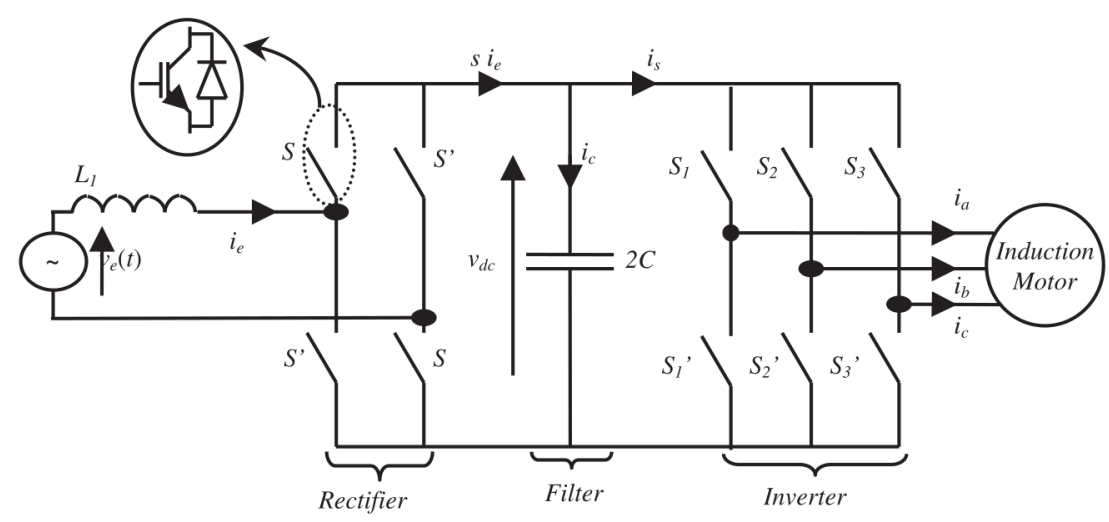

Fig. 3. The whole system under study: induction motor and associated AC/DC/AC converters.

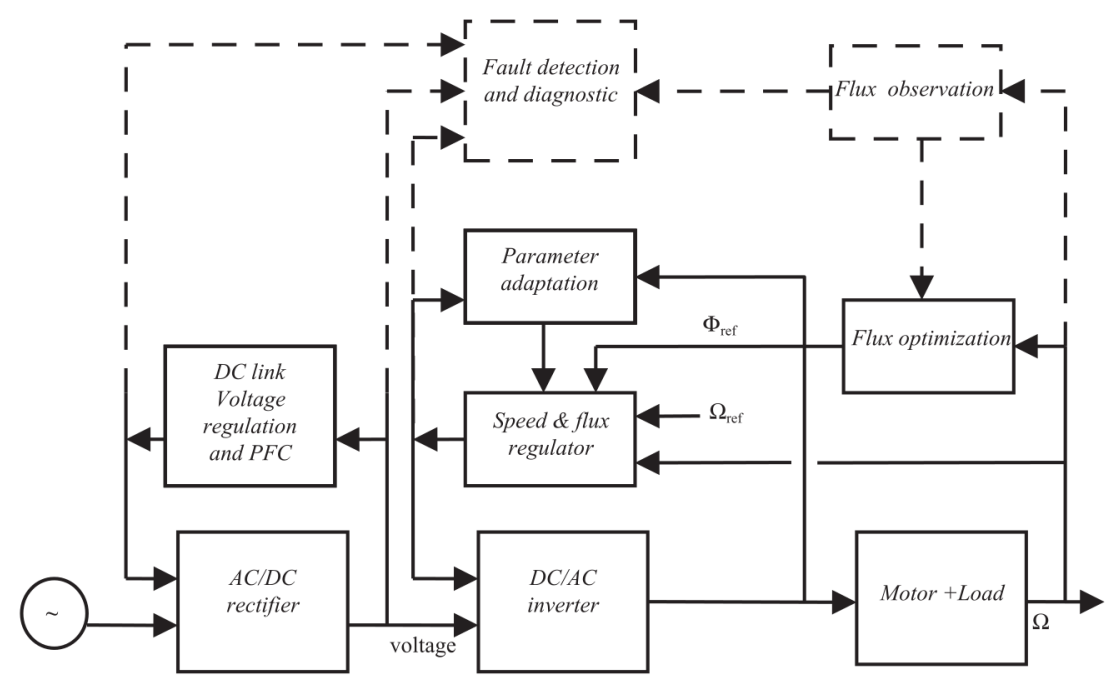

Fig. 4. Global control strategy (the dotted parts not covered in the present paper).

contributes to this purpose by proposing a theoretical framework for the system modeling, multi-objective control design and control performance analysis. Presently, the focus is made on three control objectives, i.e. wide range speed regulation, flux reference optimization and PFC. For space limitation, the issues of state observation and fault detection/diagnostic are not discussed in the present paper (Fig. 4). The first issue has already been coped with in [3] where a flux observer was developed based on the same model as presently. The second issue has been dealt with in many places, see, e.g. [20].

The control aspects dealt with in the present paper present the following features:

System modeling. The induction machine is represented by an experimentally validated model that accounts for the nonlinearity of the magnetic characteristic $[13,22]$. The power converters $(A C / D C$ converter, the $D C / A C$ inverter) are described by their respective averaged instantaneous models. There, signal averaging is resorted to cope with the binary nature of the converter switch control signals.

Control design. An adaptive multivariable controller is designed, using nonlinear techniques, e.g. backstepping control. It consists of three main components:

(i) A nonlinear bi-variable regulator is designed for the AC/DC rectifier so that the coupling between the power supply net and the rectifier operates with a unitary power factor and the connection between the rectifier and the inverter operates with a constant DC-link voltage, despite changes of the operation conditions.

(ii) An adaptive nonlinear bi-variable regulator is developed to make the motor velocity track its varying reference value and the rotor flux norm track to its optimal state-dependent reference. Parameter adaptation is resorted to cope with parameter uncertainty that characterizes especially the mechanicals parameters (load torque, rotor inertia, and friction coefficient).

(iii) An online flux reference generator is constructed that provides the speed/flux regulator with the optimal flux reference trajectory. Optimality amounts to minimize the necessary motor torque reducing thus the stator current consumption. Based on the above motor model, the optimal flux reference generator is analytically expressed in function of state variables, especially the stator currents.

Control performance analysis. The performances of the whole nonlinear adaptive controller are formally analyzed making adequate use of relevant tools from Lyapunov stability and averaging theory [6]. It will be demonstrated that all control objectives are achieved with a good accuracy. More specifically, the motor speed and rotor flux norm both track perfectly their references. Furthermore, the steady-state tracking errors associated with the rectifier input current and output voltage are shown to be harmonic signals with amplitudes depending, 
among others, on the supply net frequency. The larger the net frequency is, the smaller the tracking error amplitudes. Therefore, if the net frequency is large enough the PFC requirement will actually be guaranteed up to a harmonic error of insignificant amplitude. This formally establishes the existence of the so-called ripples (usually observed in similar practical applications) and proves why this phenomenon is generally insignificant.

The paper is organized as follows: in Section 2, the whole association including the $\mathrm{AC} / \mathrm{DC} / \mathrm{AC}$ power conversion and induction motor is modeled taking into account magnetic saturation; the multi-objective controller is designed and analyzed in Section 3; the control performances are illustrated through numerical simulations in Section 4.

\section{Modeling of the 'converter-motor' association}

The controlled system, illustrated by Fig. 3, includes an AC/DC rectifier, on one hand, and a combination 'inverter-induction motor', on the other hand. The rectifier interfaces the 'inverter-induction motor' set to the power supply net. The inverter is a DC/AC converter operating (like the $\mathrm{AC} / \mathrm{DC}$ rectifier) according to the well known Pulse Wide Modulation (PWM) principle.

\section{1. $A C / D C$ rectifier modeling}

The AC/DC rectifier is an H-bridge boost converter consisting of four IGBT's with anti-parallel diodes for bidirectional power flow mode. This is expected to accomplish two main tasks: (i) providing a constant DC link voltage and (ii) ensuring an almost unitary power factor connection (PFC) to the power supply grid. Applying Kirchhoff's laws, the rectifier can be described by the following set of differential equations:

$\frac{d i_{e}}{d t}=\frac{v_{e}}{L_{1}}-\frac{1}{L_{1}} s v_{\mathrm{dc}}$

$\frac{d v_{d c}}{d t}=\frac{1}{2 C} s i_{e}-\frac{1}{2 C} i_{s}$

where $i_{e}$ is the current in inductor $L_{1} ; v_{d c}$ denotes the voltage in capacitor 2C; $i_{s}$ designates the input current inverter, $v_{e}=\sqrt{2} \cdot E \cdot \cos \left(\omega_{e} t\right)$ is the sinusoidal net voltage (with known constants $\left.E, \omega_{e}\right)$ and $s$ is the switch binary value:

$S=\left\{\begin{array}{lll}1 & \text { if } S \text { is ON and } S^{\prime} \text { is OFF } \\ -1 & \text { if } S \text { is OFF and } S^{\prime} \text { is ON }\end{array}\right.$

The above (instantaneous) model describes accurately the physical inverter. Then, it is based upon when constructing converter simulators. However, it is not suitable for control design due to the binary nature of the control input $s$. As a matter of fact, most existing nonlinear control approaches apply to systems with continuous control inputs. Therefore, control design for the converter will be performed using the following average version of the model (1a) and (1b) [5,17]:

$$
\begin{aligned}
& \frac{d x_{1}}{d t}=\frac{v_{e}}{L_{1}}-\frac{1}{L_{1}} u_{1} x_{2} \\
& \frac{d x_{2}}{d t}=\frac{1}{2 C} u_{1} x_{1}-\frac{1}{2 C} \bar{i}_{s}
\end{aligned}
$$

where:

$x_{1}=\bar{i}_{e}, \quad x_{2}=\bar{v}_{\mathrm{dc}}, \quad u_{1}=\bar{s}$

are the average values over cutting periods of $i_{e}, v_{d c}$ and $s$, respectively.
Remark 1. In converter modeling the dead time between the two switches in one leg is ignored for this study and not considered. In other hand the standard three legs rectifiers are most used in industry. But, in traction and, more generally in the case of singlephase supply, H-bridge rectifiers are more suitable and most used.

\subsection{Inverter-motor modeling}

As mentioned in Section 1, the achievement of speed regulation and flux optimization in presence of wide range load variation necessitates that the control design is based on a model that takes into consideration the nonlinear nature of the machine magnetic circuit. Fortunately, examples of such models do exist and have been proved to be useful in control design, e.g. [15,13,22]. The model proposed in the last reference is presently preferred as, on the one hand, this model is established in the fixed $\alpha \beta$-frame and, on the other, it was experimentally validated. The experimental validation, performed on a $7.5 \mathrm{KW}$ machine, has confirmed the nonlinear nature of the corresponding magnetic characteristic (Fig. 5). The model developed in [22,13] is defined by the following physical equations:

$\frac{d \Omega}{d t}=-\frac{f}{J} \Omega+\frac{p}{J}\left(\phi_{r \alpha} i_{s \beta}-\phi_{r \beta} i_{s \alpha}\right)-\frac{T_{L}}{J}$

$\frac{d i_{s \alpha}}{d t}=-a_{2} i_{s \alpha}+\delta \phi_{r \alpha}+a_{3} p \Omega \phi_{r \beta}+a_{3} v_{s \alpha}$

$\frac{d i_{s \beta}}{d t}=-a_{2} i_{s \beta}-a_{3} p \Omega \phi_{r \alpha}+\delta \phi_{r \beta}+a_{3} v_{s \beta}$

$\frac{d \phi_{r \alpha}}{d t}=a_{1} i_{s \alpha}-L_{s e q} \delta \phi_{r \alpha}-p \Omega \phi_{r \beta}$

$\frac{d \phi_{r \beta}}{d t}=a_{1} i_{s \beta}-L_{s e q} \delta \phi_{r \beta}+p \Omega \phi_{r \alpha}$

where the various notations are defined as follows:

- $\delta$ is a varying parameter depending on the machine magnetic state as shown by Fig. 6; this dependence has been given a polynomial approximation:

$\delta=\Gamma\left(\Phi_{r}\right) \stackrel{\text { def }}{=} q_{0}+q_{1} \Phi_{r}+\cdots+q_{m} \Phi_{r}^{m}$

where the involved coefficients have been identified (based on spline approximation) using the experimental magnetic characteristic of Fig. 5.

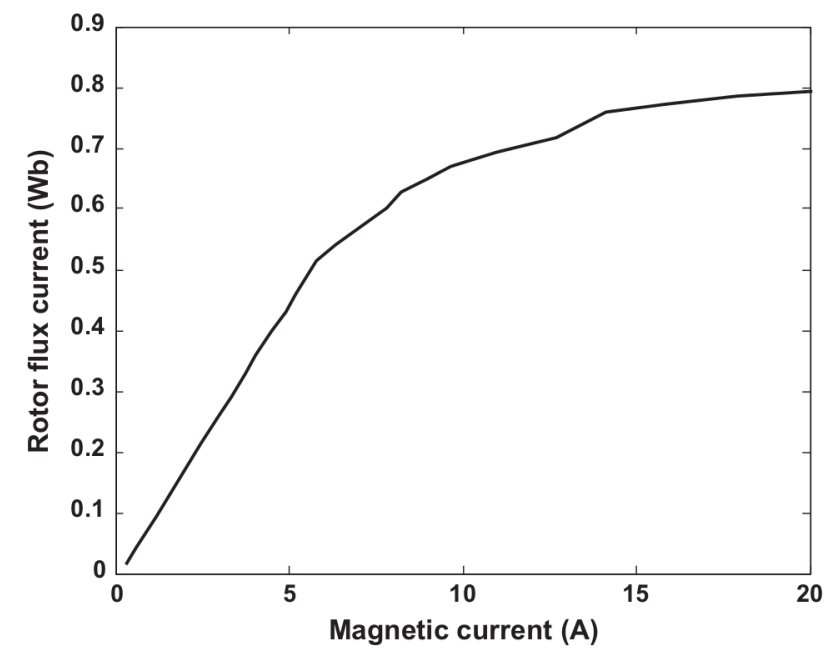

Fig. 5. Magnetic characteristic experimentally built up in [13] for a $7.5 \mathrm{KW}$ induction motor: rotor flux norm $\Phi_{r}(\mathrm{~Wb})$ versus magnetic current $I_{\mu}(\mathrm{A})$. 


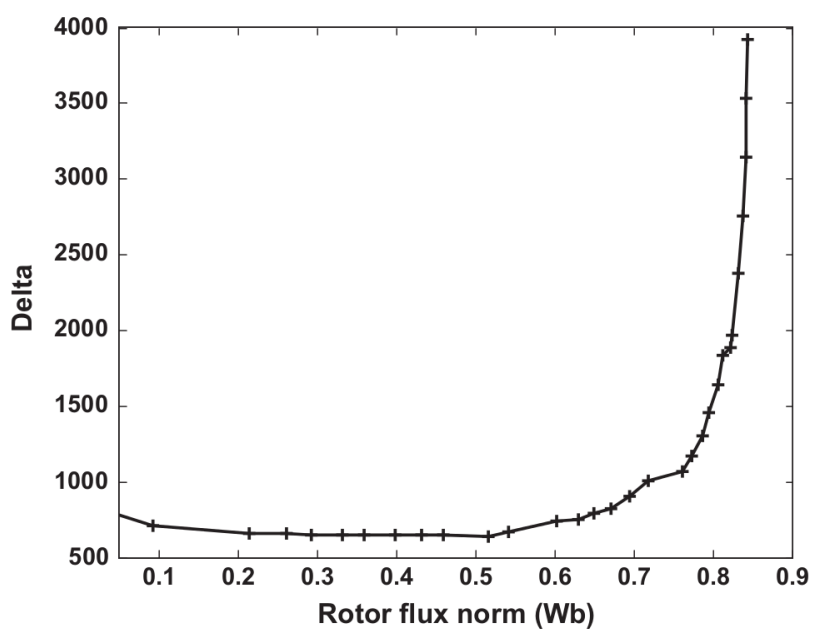

Fig. 6. Characteristic $\left(\delta, \Phi_{r}\right)$ : directly computed points $(++)$ and polynomial interpolation (solid). Unities: $\delta\left(\Omega H^{-2}\right), \Phi_{r}(\mathrm{~Wb})$.

- $\Phi_{r}$ denotes the amplitude of the (instantaneous) rotor flux, denoted $\phi_{r}$. Consequently, one has:

$$
\Phi_{r}=\sqrt{\phi_{r \alpha}^{2}+\phi_{r \beta}^{2}}
$$

where $\phi_{r \alpha}, \phi_{r \beta}$ denote the rotor flux $\alpha \beta$-components.

- $\left(i_{s \alpha}, i_{s \beta}\right)$ are the $\alpha \beta$-components of the stator current and stator voltage, respectively.

- $\Omega$ represents the motor speed.

- $R_{s}, R_{r}$ denote the stator and rotor resistances.

- $f, J, T_{L}$ are respectively the friction coefficient, rotor inertia and load torque; all three parameters are unknown.

- $p$ is the number of pole pairs.

- $L_{\text {seq }}$ is the equivalent inductance of both stator and rotor leakage brought to the stator side.

- $a_{1}=R_{r}, a_{2}=\left(R_{s}+R_{r}\right) / L_{s e q}, a_{3}=1 / L_{s e q}$.

The numerical values of the model parameters are given by Table 1 . As already mentioned, the numerical values correspond to an induction motor of $7.5 \mathrm{~kW}$.

The couple $\left(v_{s \alpha}, v_{s \beta}\right)$ denote the stator voltage in $\alpha \beta$-coordinate (Park's transformation of the three-phase stator voltages). The inverter is featured by the fact that the stator $\alpha$ - and $\beta$-voltage can be controlled independently. To this end, these voltages are expressed in function of the corresponding control action (see e.g. [10]):

$v_{s \beta}=v_{d c} u_{3}$

$v_{s \alpha}=v_{d c} u_{2}$

Table 1

Numerical values of considered motor characteristics.

\begin{tabular}{llll}
\hline Characteristic & Symbol & Value & Unit \\
\hline Nominal power & $P_{N}$ & 7.5 & $\mathrm{KW}$ \\
Nominal voltage & $U_{s n}$ & 380 & $\mathrm{~V}$ \\
Nominal flux & $\Phi_{m}$ & 0.56 & $\mathrm{~Wb}$ \\
Stator resistance & $R_{s}$ & 0.63 & $\Omega$ \\
Rotor resistance & $R_{r}$ & 0.52 & $\Omega$ \\
Inertia moment & $J$ & 0.22 & $\mathrm{~kg} \mathrm{~m}^{2}$ \\
Friction coefficient & $f$ & 0.001 & $\mathrm{~N} \mathrm{~m} \mathrm{~s} \mathrm{^{-1 }}$ \\
Number of pole pairs & $p$ & 2 & \\
Leakage equivalent inductance & $L_{s e q}$ & 7 & $\mathrm{mH}$ \\
\hline
\end{tabular}

where $\left(u_{2}, u_{3}\right)$ represent the average $\alpha$ - and $\beta$-axis components (in Park's transformation) of the three-phase duty ratio system $\left(s_{1}, s_{2}, s_{3}\right)$. The latter are defined by (1c) replacing there $S$ and $S$ by $S_{i}$ and $S_{i}^{\prime}(i=1,2,3)$. Now, let us introduce the state variables:

$x_{3}=\bar{\Omega}, \quad x_{4}=\bar{i}_{s \alpha}, \quad x_{5}=\bar{i}_{s \beta}, x_{6}=\bar{\phi}_{r \alpha}, \quad x_{7}=\bar{\phi}_{r \beta}$

where $\bar{x}$ denotes the average value over cutting periods of $x$. Then, the power absorbed by the DC/AC inverter takes the well known expression $P_{a i}=x_{2} \bar{i}_{s}$. On the other hand, the power released by the inverter is given by $P_{r m}=x_{2}\left(u_{2} x_{4}+u_{3} x_{5}\right)$. Invoking the power balance one has $P_{a i}=P_{r m}$ which yields:

$\bar{i}_{s}=\left(u_{2} x_{4}+u_{3} x_{5}\right)$

Substituting $(4 a)-(4 c)$ in (3a)-(3g) gives the following statespace representation of the association 'inverter-motor':

$$
\begin{aligned}
& \frac{d x_{3}}{d t}=-\frac{f}{J} x_{3}+\frac{p}{J}\left(x_{6} x_{5}-x_{7} x_{4}\right)-\frac{T_{L}}{J} \\
& \frac{d x_{4}}{d t}=-a_{2} x_{4}+\delta x_{6}+a_{3} p x_{3} x_{7}+a_{3} u_{2} x_{2} \\
& \frac{d x_{5}}{d t}=-a_{2} x_{5}-a_{3} p x_{3} x_{6}+\delta x_{7}+a_{3} u_{3} x_{2} \\
& \frac{d x_{6}}{d t}=a_{1} x_{4}-L_{s e q} \delta x_{6}-p x_{3} x_{7} \\
& \frac{d x_{7}}{d t}=a_{1} x_{5}-L_{s e q} \delta x_{7}+p x_{3} x_{6} \\
& \bar{\Phi}_{r}=\sqrt{x_{6}^{2}+x_{7}^{2}} \\
& \bar{\delta}=\Gamma\left(\bar{\Phi}_{r}\right)=q_{0}+q_{1} \bar{\Phi}_{r}+\cdots+q_{m} \bar{\Phi}_{r}^{m}
\end{aligned}
$$

where $\bar{\Phi}_{r}$ and $\bar{\delta}$ denote the average values over cutting periods of $\Phi_{r}$ and $\delta$ respectively. The equations obtained up to now are brought together to form the state-space model of the whole system including the $\mathrm{AC} / \mathrm{DC} / \mathrm{AC}$ converters combined with the induction motor:

$$
\begin{aligned}
& \frac{d x_{1}}{d t}=\frac{v e}{L_{1}}-\frac{1}{L_{1}} u_{1} x_{2} \\
& \frac{d x_{2}}{d t}=\frac{1}{2 C} u_{1} x_{1}-\frac{1}{2 C}\left(u_{2} x_{4}+u_{3} x_{5}\right) \\
& \frac{d x_{3}}{d t}=-\frac{f}{J} x_{3}+\frac{p}{J}\left(x_{6} x_{5}-x_{7} x_{4}\right)-\frac{T_{L}}{J} \\
& \frac{d x_{4}}{d t}=-a_{2} x_{4}+\bar{\delta} x_{6}+a_{3} p x_{3} x_{7}+a_{3} u_{2} x_{2} \\
& \frac{d x_{5}}{d t}=-a_{2} x_{5}-a_{3} p x_{3} x_{6}+\bar{\delta} x_{7}+a_{3} u_{3} x_{2} \\
& \frac{d x_{6}}{d t}=a_{1} x_{4}-L_{s e q} \bar{\delta} x_{6}-p x_{3} x_{7} \\
& \frac{d x_{7}}{d t}=a_{1} x_{5}-L_{s e q} \bar{\delta} x_{7}+p x_{3} x_{6} \\
& \bar{\Phi}_{r}=\sqrt{\left(x_{6}^{2}+x_{7}^{2}\right)} \\
& \bar{\delta}=\Gamma\left(\bar{\Phi}_{r}\right)=q_{0}+q_{1} \bar{\Phi}_{r}+\cdots+q_{m} \bar{\Phi}_{r}^{m}
\end{aligned}
$$

Recall that the load torque $T_{L}$, the rotor inertia $J$ and the friction coefficient $f$ are all unknown parameters.

\section{Controller design}

\subsection{Control objective}

Based on the whole system model (6a)-(6i), we aim at developing a controller that would be able to achieve the following objectives, despite the uncertainty that prevails on the mechanical parameters $\left(f, J, T_{L}\right)$ :

(i) Speed regulation: the machine speed $\Omega$ must track, as closely as possible, a given time-varying reference signal $\Omega_{\text {ref }}$. 
(ii) Flux optimization: the rotor flux norm $\bar{\Phi}_{r}$ must track as accurately as possible a state-dependent flux reference $\bar{\Phi}_{\text {ref }}=F\left(\bar{I}_{s}\right)$ where $I_{s}$ denotes the stator current norm and the function $F($.$) has yet to be determined so that \bar{\Phi}_{r}=\bar{\Phi}_{\text {ref }}$ entails a minimal stator current consumption.

(iii) PFC requirement: the rectifier input current $i_{e}$ must be sinusoidal and in phase or opposed phase with the AC supply voltage $v_{e}$.

As there are three control inputs at hand, namely $u_{1}, u_{2}$ and $u_{3}$, we will seek an additional control objective:

(iv) Controlling the continuous voltage $v_{d c}$ making it track a given reference signal $v_{\text {dcref }}$. The latter is generally set to a constant value equal to the nominal voltage entering the inverter.

\subsection{AC/DC rectifier control design}

\subsubsection{Controlling rectifier input current to meet PFC}

The PFC objective means that the input current rectifier should be sinusoidal and in phase (or opposite phase) with the AC supply voltage. We therefore seek a regulator that enforces the current $x_{1}$ to track a reference signal $x_{1}^{*}$ of the form:

$x_{1}^{*}=k v_{e}$

At this point $k$ is any real parameter that is allowed to be time-varying. Typically, $k$ is positive when the induction machine absorbs energy and negative when the machine restores energy (generation mode). Introduce the current tracking error:

$$
z_{1}=x_{1}-x_{1}^{*}
$$

In view of (6a), the above error undergoes the following equation:

$\dot{z}_{1}=\frac{v_{e}}{L_{1}}-\frac{1}{L_{1}} u_{1} x_{2}-\dot{x}_{1}^{*}$

To get a stabilizing control law for this first-order system, consider the quadratic Lyapunov function $V_{1}=0.5 z_{1}^{2}$. It can be easily checked that the time-derivative $\dot{V}_{1}$ is a negative definite function of $z_{1}$ if the control input is chosen to be:

$u_{1}=\frac{L_{1}\left(c_{1} z_{1}+\left(v_{e} / L_{1}\right)-\dot{x}_{1}^{*}\right)}{x_{2}}$

with $c_{1}>0$ a design parameter. The properties of such control law are summarized in the following proposition.

Proposition 1. Consider the system, next called current- or innerloop, composed of the current Eq. (6a) and the control law (10) where $c_{1}>0$ is arbitrarily chosen by the user. If the reference $x_{1}^{*}=k v_{e}$ and its first time derivative are available then one has the following properties:

(1) The current loop undergoes the equation $\dot{z}_{1}=-c_{1} z_{1}$ where $z_{1}=x_{1}-x_{1}^{*}$. As $c_{1}$ is positive this equation is globally exponentially stable, i.e. $z_{1}$ vanishes exponentially, whatever the initial conditions.

(2) If in addition $k$ converges (to a finite value), then the PFC requirement is asymptotically fulfilled in average, i.e. the (average) input current $x_{1}$ tends (exponentially fast) to its reference $k v_{e}$ as $t \rightarrow \infty$.

\subsubsection{DC link voltage regulation}

The aim is now to design a tuning law for the ratio $k$ in (7) so that the rectifier output voltage $x_{2}=\bar{v}_{d c}$ is regulated around a given reference value $v_{\text {dcref }}$. As mentioned above, $v_{\text {dcref }}$ is generally (not mandatory) chosen to be constant equal to the nominal inverter input voltage amplitude (i.e. nominal stator voltage).

$$
\text { a. Relationship between } k \text { and } x_{2} \text {. }
$$

The first step in designing such a loop is to establish the relation between the ratio $k$ (control input) and the output voltage $x_{2}$. This is the subject of the following proposition.

Proposition 2. Consider the power rectifier described by (6a) and (6b) together with the control law (10). Under the same assumptions as in Proposition 1, the output voltage $x_{2}$ varies, in response to the tuning ratio $k$, according to the equation:

$\frac{d y}{d t}=\frac{1}{C} k v_{e}^{2}+\frac{1}{C} z_{1} v_{e}+\chi(x, t)$

with $y=x_{2}^{2}$ and

$\chi(x, t)=-\frac{1}{C} x_{2}\left(u_{2} x_{4}+u_{3} x_{5}\right)$

Proof. The power absorbed by the AC/DC rectifier is given by the well known expression $P_{a b s o r b e d}=x_{1} v_{e}$. On the other hand, the power released by the rectifier (toward the load including the capacity and the inverter) is given by $P_{\text {released }}=u_{1} x_{1} x_{2}$. Power balance entails $P_{\text {absorbed }}=P_{\text {released }}$ or, equivalently:

$x_{1} v_{e}=u_{1} x_{1} x_{2}$

Also, from (7) and (8) one immediately gets that $x_{1}=k v_{e}+z_{1}$ which together with (13) yields $u_{1} x_{1}=\left(k v_{e}^{2}+z_{1} v_{e}\right) / x_{2}$. This, together with (6b), gives:

$\frac{d x_{2}}{d t}=\frac{1}{2 C x_{2}}\left(k v_{e}^{2}+z_{1} v_{e}\right)-\frac{1}{2 C}\left(u_{2} x_{4}+u_{3} x_{5}\right)$

Now, deriving $y=x_{2}^{2}$ with respect to time and using (14) yields the relation (11) and ends the proof.

\section{b. Squared DC-link voltage regulation.}

The ratio $k$ stands up as a control signal in the first-order system defined by (11). As said before, the reference signal $y_{\text {ref }} \stackrel{\text { def }}{=} v_{d c r e f}^{2}$ (of the squared DC-link voltage $x_{2}=\bar{v}_{d c}$ ) is generally given as a constant value, namely the nominal value of the inverter input voltage. Then, it follows from (11) that the tracking error $z_{2}=y-y_{\text {ref }}$ undergoes the following equation:

$\dot{z}_{2}=\frac{1}{C} E^{2} k+\frac{1}{C} E^{2} k \cos \left(2 \omega_{e} t\right)+\frac{\sqrt{2} E}{C} z_{1} \cos \left(\omega_{e} t\right)+\chi(x, t)-\dot{y}_{\text {ref }}$

where we have used the fact that $v_{e}(t)=\sqrt{2} \cdot E \cdot \cos \left(\omega_{e} t\right)$ and $v_{e}^{2}(t)=E^{2}\left(1+\cos \left(2 \omega_{e} t\right)\right)$. To get a stabilizing control law for the system (15), consider the following quadratic Lyapunov function:

$V_{2}=\frac{1}{2} z_{2}^{2}$

It is easily checked that the time-derivative $\dot{V}_{2}$ can be made negative definite in the state $z_{2}$ by letting:

$$
\begin{aligned}
& k E^{2}+k E^{2} \cos \left(2 \omega_{e} t\right)+\sqrt{2} E z_{1} \cos \left(\omega_{e} t\right) \\
& =C\left(-c_{2} z_{2}-\chi(x, t)\right)+C \dot{y}_{\text {ref }}
\end{aligned}
$$

where $c_{2}>0$ is a design parameter. The point is that such equation involves a periodic singularity due to the mutual neutralization of 
the first two terms on the left side of (17a). To get off this singularity and, besides, to avoid an excessive control chattering, we just ignore the two terms in $\cos (\cdot)$ on the left side of (17a). Therefore, we consider the following approximate and simple solution:

$k=\frac{C}{E^{2}}\left(-c_{2} z_{2}-\chi(x, t)\right)+\frac{C}{E^{2}} \dot{y}_{\text {ref }}$

Bearing in mind the fact that the first derivative of the control ratio $k$ must be available (Proposition 1 ), the following filtered version of the above solution is considered:

$\dot{k}+d k=d \frac{C}{E^{2}}\left(-c_{2} z_{2}-\chi(x, t)\right)+d \frac{C}{E^{2}} \dot{y}_{\text {ref }}$

At this point, the regulator parameters, $d$ and $c_{2}$, are any positive real constants. The forthcoming development (especially the proof of theorem 2) will make it clear how these parameters should be chosen for the control objectives to be achieved. For now, we summarize our main findings in the following proposition:

Proposition 3. Consider the control system consisting of the $A C / D C$ rectifier described by (6a) and (6b) together with the control law defined by (10) and (18). Using Proposition 1 (Part 1), it follows that the resulting closed-loop undergoes, in the $\left(z_{1}, z_{2}, k\right)$-coordinates, the following equation where $z_{1}=x_{1}-x_{1}^{*}$ and $z_{2}=y-y_{\text {ref }}$ :

$\dot{Z}_{1}=A_{1} Z_{1}+f\left(Z_{1}, t\right)+g \chi(x, t)+h \dot{y}_{r e f}^{*}$

where

$Z_{1}=\left[\begin{array}{lll}z_{1} & z_{2} & k\end{array}\right]^{T}$

$f\left(Z_{1}, t\right)=\left[0\left(b_{0} k \cos \left(2 \omega_{e} t\right)+b_{2} z_{1} \cos \left(\omega_{e} t\right)\right) 0\right]^{T} \in I R^{3}$

$A_{1}=\left(\begin{array}{ccc}-c_{1} & 0 & 0 \\ 0 & 0 & b_{0} \\ 0 & -b_{1} & -d\end{array}\right) ; g=\left[\begin{array}{ccc}0 & 1 & b_{3}\end{array}\right]^{T} ; \quad h=\left[\begin{array}{lll}0 & -1 & -b_{3}\end{array}\right]^{T}$

$b_{0}=\frac{E^{2}}{C} ; \quad b_{1}=\frac{d C}{E^{2}} c_{2} ; \quad b_{2}=\sqrt{2} \frac{E}{C} ; \quad b_{3}=-\frac{d}{E^{2}} C$

Proof. The filtering (18) makes $k$ and its derivative $k$ both available. Then, Part 1 of Proposition 1 ensures that $\dot{z}_{1}=-c_{1} z_{1}$. This, together with (15) and (18), gives the state-space equation (19a).

\subsection{Rotor flux reference optimization}

Most existing induction machine speed controllers involve a flux regulation loop (Fig. 1). Furthermore, this loop is generally designed based upon a machine standard model (ignoring the machine magnetic saturation). Then, for coherency, the flux reference signal is generally given a constant value coinciding with the machine nominal flux value $[11,14,1,21,19]$.

Of course, such controllers are unable to achieve optimal machine performances (power efficiency, power factor, maximal torque, etc.) in presence of small loads. In [15], new controllers were proposed involving a state-dependent optimal flux reference. Optimality was achieved in the sense of stator current consumption, using models that account for the magnetic circuit saturation. Presently, a similar flux reference optimality is sought but, unlike the previous references, the machine mechanical parameters and load torque are subject to uncertainty and the control strategy accounts for the presence of power conversion elements (Fig. 4).
In this subsection, the aim is to find a relationship between the rotor flux norm and the stator current norm. In this respect, let us recall that the norms of electrical quantities are invariant under frame Park change. Then, it makes sense, for simplicity reasons, to perform all this section computations in the Park oriented $d-q$ reference frame. Indeed, within this reference frame, the flux q-component is (or can be considered to be) null and all state variables are constant in steady-state. Then, the machine electromagnetic torque $T_{e}$ is expressed as follows:

$T_{e}=p \phi_{r d} i_{s q}=p \Phi_{r} i_{s q}$

On the other hand, Eq. (3d) becomes in the Park oriented $d-q$ reference frame:

$\frac{d \Phi_{r}}{d t}=a_{1} i_{s d}-L_{s e q} \delta \Phi_{r}$

and that the steady-state current $i_{s d}$ can be given the following expression:

$i_{s d}=\frac{L_{s e q}}{a_{1}} \delta \Phi_{r}$

In (20), (21a) and (21b), $i_{s d}$ and $i_{s q}$ denote the $d$ - and $q$-components of the stator current. Also, the stator current norm expression simplifies to:

$I_{s}^{2}=i_{s d}^{2}+i_{s q}^{2}$

Then, using (20)-(22), one gets the following expression of the electromagnetic torque:

$T_{e}=p \Phi_{r} \sqrt{I_{s}^{2}-\left(\frac{L_{s e q}}{a_{1}} \delta \Phi_{r}\right)^{2}}$

Fig. 7 shows the curves representing the electromagnetic torque $T_{e}$ versus the flux $\Phi_{r}$, for various values of the stator current $I_{s}$. It is clearly seen that for a given torque there are many operation points that only differ by the value of the flux $\Phi_{r}$ and the current $I_{s}$. For instance, a torque $T_{e}=20 \mathrm{Nm}$ can be produced, for the machine of Table 1 , with:

- A flux $\Phi_{r}=0.50 \mathrm{~Wb}$ and a current $I_{S}=8.5 \mathrm{~A}$.

- A flux $\Phi_{r}=0.37 \mathrm{~Wb}$ and a current $I_{s}=10 \mathrm{~A}$.

- A flux $\Phi_{r}=0.21 \mathrm{~Wb}$ and a current $I_{s}=16 \mathrm{~A}$.

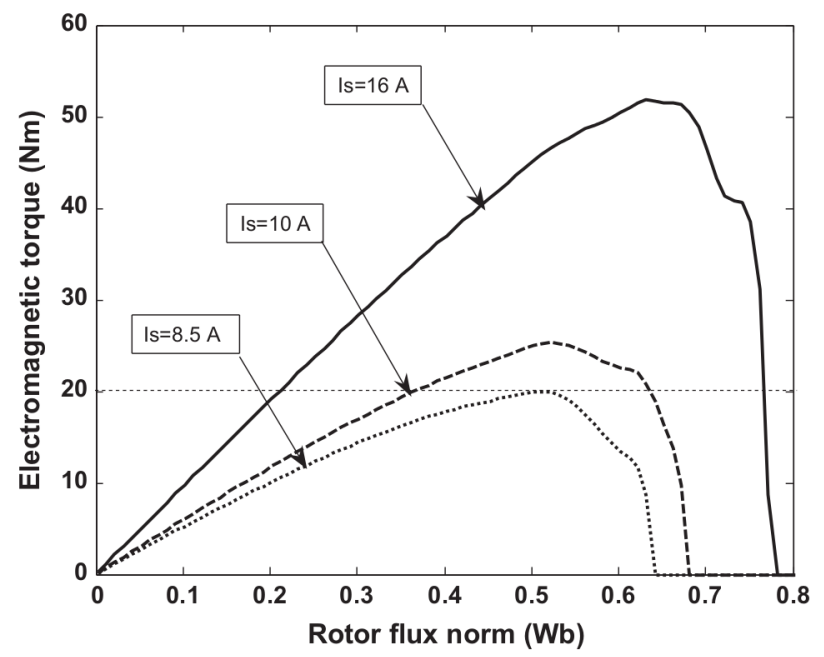

Fig. 7. Electromagnetic torque $T_{e}(\mathrm{Nm})$ in function of rotor flux $\Phi_{r}$, for different stator currents norm $I_{s}$ 
From an energetic viewpoint, the best operation point is one that involves the least current consumption. Let $T_{e i}(i=1, \ldots, r)$ be a sufficiently large sample of relevant torque values. It is readily seen from Fig. 7 that for any $T_{e i}$, there is a unique couple $\left(\Phi_{r i}, I_{s i}\right)$ that involves the least possible stator current. A set of couples $\left(\Phi_{r i}, I_{s i}\right)$ has thus been obtained using (23) and interpolated to get a polynomial function $F(\cdot)$ such that $\Phi_{r i}=F\left(I_{s i}\right)$. The polynomial interpolation thus constructed is denoted:

$F\left(I_{s}\right)=h_{n} I_{s}^{n}+h_{n-1} I_{s}^{n-1}+\cdots+h_{1} I_{s}^{+} h_{0}$

Such polynomial function is referred to optimal current-flux (OCF) characteristic and is illustrated by Fig. 8. Recall that optimality is understood in the sense of minimal absorbed stator current $I_{s}$ for a given torque $T_{e}$.

Remark 2. The polynomial interpolation yielding the function $F(\cdot)$ has been obtained the Matlab functions POLYDER, POLYVAL, SPLINE, and POLYFIT.

\subsection{Speed and flux adaptive control design and analysis}

In this section, a speed and flux adaptive regulator is designed for the saturated induction machine described by (6c)-(6i). The speed reference $\Omega_{\text {ref }}$ is any bounded and derivable function of time and its two first derivatives are available and bounded. These requirements can always be met by filtering the reference through second-order linear filters. The flux reference $\bar{\Phi}_{\text {ref }}$ is obtained online from the average value of the stator current so that, if $\bar{\Phi}_{r}=\bar{\Phi}_{\text {ref }}$ (with $\bar{\Phi}_{r}=\sqrt{x_{6}^{2}+x_{7}^{2}}$ ), the machine will operate in optimal conditions (i.e. with minimal absorbed stator current). In the light of Section 3.3, it follows that such optimality will be guaranteed if the flux reference is computed online as follows:

$\bar{\Phi}_{\text {ref }}=F\left(\bar{I}_{s}\right)=h_{n} \bar{I}_{\mathrm{s}}^{n}+h_{n-1} \bar{I}_{\mathrm{s}}^{n-1}+\cdots+h_{1} \bar{I}_{s}+h_{0}$

In fact, the relation (25) is nothing other than (24) replacing there the variables by their average values (because the system model (6a)-(6i) involves average variables).

The adaptive speed/flux controller design will now be performed in two steps using the backstepping technique [7]. First, introduce the tracking errors:

$z_{3}=\Omega_{\text {ref }}-x_{3}$

$z_{4}=\bar{\Phi}_{r e f}^{2}-\left(x_{6}^{2}+x_{7}^{2}\right)$

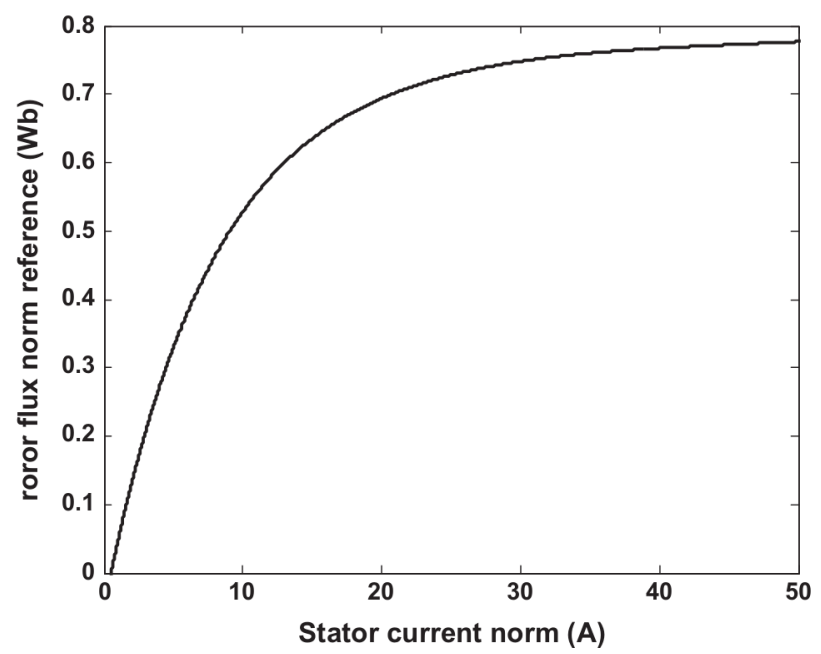

Fig. 8. Optimal current-flux characteristic obtained from the polynomial interpolation of the experimental points $\left(\Phi_{T_{e_{j}}}^{*}, I_{T_{e_{j}}}^{*}\right)$ for the induction machine with physical characteristics of Table 1 .
Step 1 It follows from (5a), (5d), and (5e) that the errors $z_{3}$ and $z_{4}$ undergo the differential equations:

$$
\begin{aligned}
\dot{z}_{3}= & \dot{\Omega}_{\text {ref }}-\frac{p}{J}\left(x_{6} x_{5}-x_{7} x_{4}\right)+\frac{T_{L}}{J}+\frac{f}{J} x_{3} \\
\dot{z}_{4}= & 2 \bar{\Phi}_{\text {ref }} \dot{\bar{\Phi}}_{\text {ref }}-2 x_{6} \dot{x}_{6}-2 x_{7} \dot{x}_{7} \\
= & 2 \bar{\Phi}_{\text {ref }} \dot{\bar{\Phi}}_{\text {ref }}-2 x_{6}\left(a_{1} x_{4}-L_{\text {seq }} \bar{\delta} x_{6}-p x_{3} x_{7}\right)-2 x_{7}\left(a_{1} x_{5}\right. \\
& \left.-L_{\text {seq }} \bar{\delta}_{7}+p x_{3} x_{6}\right) \\
= & 2 \bar{\Phi}_{\text {ref }} \dot{\bar{\Phi}}_{\text {ref }}-2 a_{1}\left(x_{6} x_{4}+x_{7} x_{5}\right)+2 L_{\text {seq }} \bar{\delta}\left(x_{6}^{2}+x_{7}^{2}\right) \\
\dot{z}_{4}= & 2 \bar{\Phi}_{\text {ref }} \dot{\bar{\Phi}}_{\text {ref }}-2 a_{1}\left(x_{6} x_{4}+x_{7} x_{5}\right)+2 L_{\text {seq }} \bar{\delta}\left(\bar{\Phi}_{r e f}^{2}-z_{4}\right)
\end{aligned}
$$

In (27) and (28), the quantities $p\left(x_{6} x_{5}-x_{7} x_{4}\right)$ and $2 a_{1}\left(x_{6} x_{4}+x_{7} x_{5}\right)$ stand up as virtual control signals. Let us temporarily suppose these to be the actual control signals and consider the Lyapunov function candidate:

$V_{3}=\frac{1}{2}\left(z_{3}^{2}+z_{4}^{2}\right)$

It can be easily checked that the time derivative of (29) can be made negative definite function of $\left(z_{3}, z_{4}\right)$, i.e.

$\dot{V}_{3}=-c_{3} z_{3}^{2}-c_{4} z_{4}^{2}$

by letting $p\left(x_{6} x_{5}-x_{7} x_{4}\right)=\mu_{1}$ and $2 a_{1}\left(x_{6} x_{4}+x_{7} x_{5}\right)=v_{1}$ with:

$\mu_{1} \stackrel{\text { def }}{=} J\left(c_{3} z_{3}+\dot{\Omega}_{r e f}\right)+T_{L}+f\left(\Omega_{r e f}-z_{3}\right)$

and

$v_{1} \stackrel{\text { def }}{=} c_{4} z_{4}+2 \bar{\Phi}_{\text {ref }} \dot{\bar{\Phi}}_{\text {ref }}+2 L_{\text {seq }} \bar{\delta}\left(\bar{\Phi}_{\text {ref }}^{2}-z_{4}\right)$

where $c_{3}$ and $c_{4}$ are any positive design parameters. Since $J, T_{L}$ and $f$ are unknown, the first equation in (31) is replaced by its certainty equivalence from, yielding the following adaptive control laws:

$\mu_{1} \stackrel{\text { def }}{=} \widehat{J}\left(c_{3} z_{3}+\dot{\Omega}_{\text {ref }}\right)+\widehat{T}_{L}+\hat{f}\left(\Omega_{r e f}-z_{3}\right)$

$v_{1} \stackrel{\text { def }}{=} c_{4} z_{4}+2 \bar{\Phi}_{\text {ref }} \dot{\bar{\Phi}}_{\text {ref }}+2 L_{\text {seq }} \bar{\delta}\left(\bar{\Phi}_{\text {ref }}^{2}-z_{4}\right)$

and $\widehat{J}, \widehat{T}_{L}$ and $\hat{f}$ are estimates (yet to be determined) of $J, T_{L}$ and $f$, respectively. As the quantities $p\left(x_{6} x_{5}-x_{7} x_{4}\right)$ and $2 a_{1}\left(x_{6} x_{4}+x_{7} x_{5}\right)$ are not the actual control signals, they cannot be let equal to $\mu_{1}$ and $v_{1}$, respectively. Nevertheless, we retain the expressions of $\mu_{1}$ and $v_{1}$ as the first stabilizing functions and introduce the new errors:

$z_{5}=\mu_{1}-p\left(x_{6} x_{5}-x_{7} x_{4}\right)$

$z_{6}=v_{1}-2 a_{1}\left(x_{6} x_{4}+x_{7} x_{5}\right)$

Then, using the notations (29)-(34), the dynamics of the errors $z_{3}$ and $z_{4}$, initially described by (27)-(30), can be rewritten as follows:

$$
\begin{aligned}
& \dot{z}_{3}=\dot{\Omega}_{r e f}-\frac{1}{J}\left(\mu_{1}-z_{5}\right)+\frac{T_{L}}{J}+\frac{f}{J} x_{3} \\
& \dot{z}_{3}=\dot{\Omega}_{r e f}-\frac{1}{J}\left[\widehat{J}\left(c_{3} z_{3}+\dot{\Omega}_{r e f}\right)+\widehat{T}_{L}+\hat{f} x_{3}-z_{5}\right]+\frac{T_{L}}{J}+\frac{f}{J} x_{3} \\
& \dot{z}_{3}=-c_{3} z_{3}+\frac{\widetilde{J}}{J}\left(c_{3} z_{3}+\dot{\Omega}_{r e f}\right)+\frac{\widetilde{T}_{L}}{J}+\frac{\tilde{f}}{J} x_{3}+\frac{1}{J} z_{5} \\
& \dot{z}_{4}=-c_{4} z_{4}+z_{6}
\end{aligned}
$$

where

$\widetilde{J}=J-\widehat{J}, \quad \widetilde{T}_{L}=T_{L}-\widehat{T}_{L} \quad$ and $\quad \tilde{f}=f-\hat{f}$ 
Similarly, the time-derivative of $V_{3}$ can be expressed in function of the new errors as follows:

$$
\begin{aligned}
\dot{V}_{3}= & -c_{3} z_{3}^{2}-c_{4} z_{4}^{2}+z_{4} z_{6} \\
& +z_{3}\left[\frac{\widetilde{J}}{J}\left(c_{3} z_{3}+\dot{\Omega}_{\text {ref }}\right)+\frac{\widetilde{T}_{L}}{J}+\frac{\tilde{f}}{J} x_{3}+\frac{1}{J} z_{5}\right]
\end{aligned}
$$

Step 2: The second design step consists in choosing the actual control signals, $u_{2}$ and $u_{3}$, so that all errors $\left(z_{3}, z_{4}, z_{5}, z_{6}\right)$ converge to zero. To this end, it must be made clear how these errors depend on the actual control signals $\left(u_{2}, u_{3}\right)$. First, focusing on $z_{5}$, it follows from (33) that:

$\dot{z}_{5}=\dot{\mu}_{1}-p\left(\dot{x}_{6} x_{5}+x_{6} \dot{x}_{5}-\dot{x}_{7} x_{4}-x_{7} \dot{x}_{4}\right)$

Using (5a)-(5e), (35c) and (31), one gets from (37):

$$
\begin{aligned}
\dot{z}_{5}= & {\left[\hat{J}\left(c_{3} \dot{z}_{3}+\ddot{\Omega}_{r e f}\right)-\dot{\tilde{J}}\left(c_{3} z_{3}+\dot{\Omega}_{r e f}\right)-\dot{\widetilde{T}}_{L}-\dot{\tilde{f}} x_{3}+\hat{f} \dot{x}_{3}\right] } \\
& -p\left(\dot{x}_{6} x_{5}-\dot{x}_{7} x_{4}\right)-p\left(x_{6} \dot{x}_{5}-x_{7} \dot{x}_{4}\right)
\end{aligned}
$$

$$
\dot{z}_{5}=\left(c_{3} \widehat{J}-\hat{f}\right)\left[-c_{3} z_{3}+\frac{\tilde{J}}{J}\left(c_{3} z_{3}+\dot{\Omega}_{r e f}\right)+\frac{\tilde{T}_{L}}{J}+\frac{\tilde{f}}{J} x_{3}+\frac{1}{J} z_{5}\right]
$$$$
+\widehat{\jmath} \ddot{\Omega}_{r e f}+\hat{f} \dot{\Omega}_{r e f}-p\left(\left(a_{1} x_{4}-L_{s e q} \bar{\delta} x_{6}-p x_{3} x_{7}\right) x_{5}-\left(a_{1} x_{5}\right.\right.
$$$$
\left.\left.-L_{\text {seq }} \bar{\delta} x_{7}+p x_{3} x_{6}\right) x_{4}\right)-\left[\dot{\tilde{J}}\left(c_{3} z_{3}+\dot{\Omega}_{r e f}\right)+\dot{\tilde{T}}_{L}+\dot{\tilde{f}} x_{3}\right]
$$$$
-p x_{6}\left(-a_{2} x_{5}-a_{3} p x_{3} x_{6}+\bar{\delta} x_{7}+a_{3} u_{3} x_{2}\right)+p x_{7}\left(-a_{2} x_{4}\right.
$$$$
\left.+\bar{\delta} x_{6}+a_{3} p x_{3} x_{7}+a_{3} u_{2} x_{2}\right)
$$

For convenience, the above equation is given the following compact form:

$$
\begin{aligned}
& \dot{z}_{5}=\mu_{2}+\frac{c_{3} \widehat{J}-\hat{f}}{J} z_{5}+p a_{3} x_{2}\left(x_{7} u_{2}-x_{6} u_{3}\right) \\
& +\left(c_{3} \widehat{J}-\hat{f}\right)\left[\frac{\tilde{J}}{J}\left(c_{3} z_{3}+\dot{\Omega}_{r e f}\right)+\frac{\widetilde{T}_{L}}{J}+\frac{\tilde{f}}{J} \chi_{3}\right] \\
& -\left[\dot{\widetilde{J}}\left(c_{3} z_{3}+\dot{\Omega}_{r e f}\right)+\dot{\widetilde{T}}_{L}+\dot{\tilde{f}} x_{3}\right]
\end{aligned}
$$

with

$$
\begin{aligned}
\mu_{2}= & {\left[-c_{3} z_{3}\left(c_{3} \hat{J}-\hat{f}\right)+\widehat{J} \ddot{Q}_{r e f}+\hat{f} \dot{\Omega}_{r e f}\right]+p^{2} a_{3} x_{3}\left(x_{6}^{2}+x_{7}^{2}\right) } \\
& +p\left(L_{s e q} \bar{\delta}+a_{2}\right)\left(x_{6} x_{5}-x_{7} x_{4}\right)+p^{2} x_{3}\left(x_{7} x_{5}+x_{6} x_{4}\right)
\end{aligned}
$$

Similarly, it follows from (34) that, $z_{6}$ undergoes the following differential equation:

$\dot{z}_{6}=\dot{v}_{1}-2 a_{1}\left(\dot{x}_{6} x_{4}+x_{6} \dot{x}_{4}+\dot{x}_{7} x_{5}+x_{7} \dot{x}_{5}\right)$

Using (5a)-(5e) and (32b), it follows from (41):

$$
\begin{aligned}
\dot{z}_{6}= & c_{4} \dot{z}_{4}+2 \bar{\Phi}_{\text {ref }} \ddot{\bar{\Phi}}_{\text {ref }}+2 \dot{\bar{\Phi}}_{\text {ref }}^{2}+2 L_{\text {seq }} \bar{\delta}\left(2 \bar{\Phi}_{r e f} \dot{\bar{\Phi}}_{\text {ref }}-\dot{z}_{4}\right) \\
& +2 L_{s e q} \dot{\bar{\delta}}\left(\bar{\Phi}_{r e f}^{2}-z_{4}\right)-2 a_{1} x_{4}\left(a_{1} x_{4}-L_{\text {seq }} \bar{\delta} x_{6}-p x_{3} x_{7}\right) \\
& -2 a_{1} x_{6}\left(-a_{2} x_{4}+\bar{\delta} x_{6}+a_{3} p x_{3} x_{7}+a_{3} u_{2} x_{2}\right) \\
& -2 a_{1} x_{5}\left(a_{1} x_{5}-L_{s e q} \bar{\delta} x_{7}+p x_{3} x_{6}\right) \\
& -2 a_{1} x_{7}\left(-a_{2} x_{5}-a_{3} p x_{3} x_{6}+\bar{\delta} x_{7}+a_{3} u_{3} x_{2}\right)
\end{aligned}
$$

where the derivative of $\bar{\delta}$ is obtained from (6i):

$\dot{\bar{\delta}}=\frac{d \bar{\delta}}{d \bar{\Phi}_{r}} \frac{d \bar{\Phi}_{r}}{d t}=\frac{d \bar{\delta}}{d \bar{\Phi}_{r}}\left(\frac{x_{6}}{\bar{\Phi}_{r}} \dot{x}_{6}+\frac{x_{7}}{\bar{\Phi}_{r}} \dot{x}_{7}\right)$

Eq. (34) is in turn given the following compact form:

$\dot{z}_{6}=v_{2}-2 a_{1} a_{3} x_{2}\left(x_{6} u_{2}+x_{7} u_{3}\right)$ with

$$
\begin{aligned}
v_{2}= & c_{4} \dot{z}_{4}+2 \bar{\Phi}_{r e f} \ddot{\bar{\Phi}}_{\text {ref }}+2 \dot{\bar{\Phi}}_{\text {ref }}^{2}+2 L_{\text {seq }} \bar{\delta}\left(2 \bar{\Phi}_{\text {ref }} \dot{\bar{\Phi}}_{\text {ref }}-\dot{z}_{4}\right) \\
& +2 L_{\text {seq }} \dot{\bar{\delta}}\left(\bar{\Phi}_{\text {ref }}^{2}-z_{4}\right)-2 a_{1} x_{4}\left(a_{1} x_{4}-L_{\text {seq }} \bar{\delta} x_{6}-p x_{3} x_{7}\right) \\
& -2 a_{1} x_{6}\left(-a_{2} x_{4}+\bar{\delta} x_{6}+a_{3} p x_{3} x_{7}\right) \\
& -2 a_{1}\left(a_{1} x_{5}-L_{\text {seq }} \bar{\delta} x_{7}+p x_{3} x_{6}\right) x_{5} \\
& -2 a x_{7}\left(-a_{2} x_{5}-a_{3} p x_{3} x_{6}+\bar{\delta} x_{7}\right) \\
v_{2}= & \left(c_{4}-2 L_{\text {seq }} \bar{\delta}\right)\left(-c_{4} z_{4}+z_{6}\right)+2 \bar{\Phi}_{r e f} \ddot{\bar{\Phi}}_{\text {ref }}+2 \dot{\bar{\Phi}}_{\text {ref }}^{2} \\
& +4 L_{\text {seq }} \bar{\delta} \bar{\Phi}_{r e f} \dot{\bar{\Phi}}_{r e f}+2 L_{s e q} \dot{\bar{\delta}}\left(\bar{\Phi}_{r e f}^{2}-z_{4}\right)+2 a_{1} x_{3} p\left(x_{7} x_{4}\right. \\
& \left.-x_{5} x_{6}\right)-2 a_{1} \bar{\delta}\left(\bar{\Phi}_{r e f}^{2}-z_{4}\right)+2 a_{1}\left(L_{s e q} \bar{\delta}+a_{2}\right)\left(x_{4} x_{6}\right. \\
& \left.+x_{5} x_{7}\right)-2\left(a_{1}\right)^{2}\left(x_{4}^{2}+x_{5}^{2}\right)
\end{aligned}
$$

To analyze the stability of the error system, composed of equations (35a), (35b), (39) and (44a), consider the following augmented Lyapunov function candidate:

$V_{4}=\frac{1}{2} z_{3}^{2}+\frac{1}{2} z_{4}^{2}+\frac{1}{2} z_{5}^{2}+\frac{1}{2} z_{6}^{2}+\frac{1}{2} \frac{\widetilde{J}^{2}}{J}+\frac{1}{2} \frac{\widetilde{T}_{L}^{2}}{J}+\frac{1}{2} \frac{\tilde{f}^{2}}{J}$

Its time-derivative along the trajectory of the state vector $\left(z_{3}, z_{4}, z_{5}, z_{6}\right)$ is:

$\dot{V}_{4}=z_{3} \dot{z}_{3}+z_{4} \dot{z}_{4}+z_{5} \dot{z}_{5}+z_{6} \dot{z}_{6}+\frac{\tilde{J} J}{J}+\frac{\tilde{f} \dot{\tilde{f}}}{J}+\frac{\widetilde{T}_{L} \dot{\tilde{T}}_{L}}{J}$

Using (36), (39) and (44a), Eq. (46) gives:

$$
\begin{aligned}
\dot{V}_{4}= & z_{3}\left[-c_{3} z_{3}+\frac{\tilde{J}}{J}\left(c_{3} z_{3}+\dot{\Omega}_{r e f}\right)+\frac{\widetilde{T}_{L}}{J}+\frac{\tilde{f}}{J} x_{3}+\frac{1}{J} z_{5}\right] \\
& +z_{4}\left[-c_{4} z_{4}+z_{6}\right] \\
& +z_{5}\left(c_{3} \hat{J}-\hat{f}\right)\left(\frac{\tilde{J}}{J}\left(c_{3} z_{3}+\dot{\Omega}_{r e f}\right)+\frac{\widetilde{T}_{L}}{J}+\frac{\tilde{f}}{J} x_{3}\right)+z_{5}\left[\mu_{2}\right. \\
& \left.+c_{3} z_{5}+p a_{3} x_{2}\left(x_{7} u_{2}-x_{6} u_{3}\right)\right]+z_{6}\left(v_{2}-2 a_{1} x_{2} a_{3}\left(x_{7} u_{3}\right.\right. \\
& \left.\left.+x_{6} u_{2}\right)\right)+z_{5}\left[-c_{3} z_{5} \frac{\tilde{J}}{J}-\frac{f}{J} z_{5}+z_{5} \frac{\tilde{f}}{J}\right]+\frac{\widetilde{J}}{\tilde{J}}+\frac{\widetilde{T}_{L}}{J} \dot{\widetilde{T}}_{L}+\frac{\tilde{f}}{\tilde{J}} \\
& -z_{5}\left[\dot{\tilde{J}}\left(c_{3} z_{3}+\dot{\Omega}_{r e f}\right)+\dot{\widetilde{T}}_{L}+\dot{\tilde{f}} x_{3}\right]
\end{aligned}
$$

Adding $c_{5} z_{5}^{2}-c_{5} z_{5}^{2}+c_{6} z_{6}^{2}-c_{6} z_{6}^{2}$ to the right side of (47a) and rearranging terms, yields:

$$
\begin{aligned}
\dot{V}_{4}= & -c_{3} z_{3}^{2}-c_{4} z_{4}^{2}-c_{5} z_{5}^{2}-c_{6} z_{6}^{2}-\frac{f}{J} z_{5}^{2}+\frac{1}{J} z_{3} z_{5} \\
& +\frac{\widetilde{J}}{J}\left[\dot{\tilde{J}}+\left(c_{3} z_{3}+\dot{\Omega}_{r e f}\right) z_{3}+z_{5}\left(c_{3} \widehat{J}-\hat{f}\right)\left(c_{3} z_{3}+\dot{\Omega}_{\text {ref }}\right)-c_{3} z_{5}^{2}\right] \\
& +\widetilde{T}_{L}\left[\dot{\tilde{T}}_{L}+z_{3}+z_{5}\left(c_{3} \widehat{J}-\hat{f}\right)\right]+\frac{\tilde{f}}{J}\left[\dot{\tilde{f}}+z_{3} x_{3}+z_{5} x_{3}\left(c_{3} \widehat{J}-\hat{f}\right)+z_{5}^{2}\right] \\
& +z_{5}\left[\mu_{2}+\left(c_{5}+c_{3}\right) z_{5}+p a_{3} x_{2}\left(x_{7} u_{2}-x_{6} u_{3}\right)\right] \\
& -z_{5}\left[\dot{\tilde{J}}\left(c_{3} z_{3}+\dot{\Omega}_{\text {ref }}\right)+\dot{\tilde{T}}_{L}+\dot{\tilde{f}} x_{3}\right] \\
& +z_{6}\left(z_{4}+v_{2}+c_{6} z_{6}-2 a_{1} x_{2} a_{3}\left(x_{7} u_{3}+x_{6} u_{2}\right)\right)
\end{aligned}
$$

which suggests the following parameter adaptation laws:

$\dot{\tilde{T}}_{L}=-\lambda_{T_{L}}, \quad \dot{\tilde{J}}=-\lambda_{J} \quad$ and $\quad \dot{\tilde{f}}=-\lambda_{f}$

with

$$
\begin{aligned}
& \lambda_{J}=-c_{3} z_{5}^{2}+z_{3}\left(c_{3} z_{3}+\dot{\Omega}_{r e f}\right)+z_{5}\left(c_{3} \widehat{J}-\hat{f}\right)\left(c_{3} z_{3}+\dot{\Omega}_{\text {ref }}\right) \\
& \lambda_{T_{L}}=z_{3}+\left(c_{3} \widehat{J}-\hat{f}\right) z_{5} \\
& \lambda_{f}=z_{3} x_{3}+z_{5} x_{3}\left(c_{3} \widehat{J}-\hat{f}\right)+z_{5}^{2}
\end{aligned}
$$


Substituting the parameter adaptation laws (47c) to $\dot{\widetilde{T}}_{L}, \dot{\tilde{J}}$ and $\dot{\tilde{f}}$ in the right side of (47b) yields:

$$
\begin{aligned}
\dot{V}_{4}= & -c_{3} z_{3}^{2}-c_{4} z_{4}^{2}-c_{5} z_{5}^{2}-c_{6} z_{6}^{2}-\frac{f}{J} z_{5}^{2}+\frac{1}{J} z_{3} z_{5}+z_{5}\left[\mu_{2}+\left(c_{5}\right.\right. \\
& \left.\left.+c_{3}\right) z_{5}+p a_{3} x_{2}\left(x_{7} u_{2}-x_{6} u_{3}\right)\right] \\
& +z_{5}\left(\lambda_{J}\left(c_{3} z_{3}+\dot{\Omega}_{r e f}\right)+\lambda_{T_{L}}+\lambda_{f} x_{3}\right)+z_{6}\left[v_{2}+z_{4}+c_{6} z_{6}\right. \\
& \left.-2 a_{1} a_{3} x_{2}\left(x_{6} u_{2}+x_{7} u_{3}\right)\right]
\end{aligned}
$$

where $c_{5}$ and $c_{6}$ are new arbitrary positive real design parameters. Eq. (48) suggests that the control signals $u_{2}, u_{3}$ should be chosen in order to set to zero the two quantities between curly brackets (on the right side of (48)). Letting these quantities equal to zero and solving the resulting second-order linear equation system with respect to $\left(u_{2}, u_{3}\right)$, gives the following control law:

$$
\left[\begin{array}{l}
u_{2} \\
u_{3}
\end{array}\right]=\left[\begin{array}{ll}
\lambda_{0} & \lambda_{1} \\
\lambda_{2} & \lambda_{3}
\end{array}\right]^{-1}\left[\begin{array}{l}
U_{A} \\
U_{B}
\end{array}\right]
$$

with:

$$
\begin{aligned}
& U_{A}=-\left(\lambda_{J}\left(c_{3} z_{3}+\dot{\Omega}_{r e f}\right)+\lambda_{T_{L}}+\lambda_{f} x_{3}\right)-\left(c_{5}+c_{3}\right) z_{5}-\mu_{2} \\
& U_{B}=-z_{4}-c_{6} z_{6}-v_{2}, \quad \lambda_{0}=p a_{3} x_{7} x_{2}, \quad \lambda_{1}=-p a_{3} x_{6} x_{2}, \\
& \lambda_{2}=-2 a_{1} a_{3} x_{6} x_{2}, \quad \lambda_{3}=-2 a_{1} a_{3} x_{7} x_{2}
\end{aligned}
$$

Note that inversed matrix in (49) is nonsingular in practice because its determinant is given by $D=\lambda_{0} \lambda_{3}-\lambda_{1} \lambda_{2}=$ $-2 p a_{1}^{2} a_{3} x_{2}^{2}\left(x_{6}^{2}+x_{7}^{2}\right)$ and $\sqrt{x_{6}^{2}+x_{7}^{2}}=\bar{\Phi}_{r}$ never vanish in practice because of the machine nonzero remnant flux and the small variations of $x_{2}=v_{d c}$ with respect to its high nominal value $(600 \mathrm{~V})$. Finally, supposing the unknown parameters $\left(T_{L}, J, f\right)$ be constant values, one gets from (35c) and (47c)-(47f), the following parameter adaptive laws:

$\dot{\widehat{J}}=-c_{3} z_{5}^{2}+z_{3}\left(c_{3} z_{3}+\dot{\Omega}_{r e f}\right)+z_{5}\left(c_{3} \widehat{J}-\hat{f}\right)\left(c_{3} z_{3}+\dot{\Omega}_{\text {ref }}\right)$

$\dot{\hat{T}}_{L}=z_{3}+\left(c_{3} \hat{J}-\hat{f}\right) z_{5}$

$\dot{\hat{f}}=z_{3} x_{3}+z_{5} x_{3}\left(c_{3} \widehat{J}-\hat{f}\right)+z_{5}^{2}$

The properties of the speed/flux regulator thus designed are described in the following proposition:

Theorem 1 (Speed/flux regulator). Consider the closed-loop system composed of the induction machine described by the model (5), supposing there the unknown parameters $\left(T_{L}, J, f\right)$ to be constant, and the nonlinear adaptive regulator defined by the control law (49), the parameter update laws (51)-(53) and the flux generator (25). Then, one has the following properties:

(1) The closed-loop error system undergoes the following equations, in the $\left(z_{3}, z_{4}, z_{5}, z_{6}\right)$ coordinates:

$$
\begin{aligned}
& \dot{z}_{3}=-c_{3} z_{3}+\frac{1}{J} z_{5}+\zeta_{3}\left(z_{3}, z_{5}, \widetilde{\Theta}, t\right) \\
& \dot{z}_{4}=-c_{4} z_{4}+z_{6} \\
& \dot{z}_{5}=-\left(c_{5}+\frac{f}{J}\right) z_{5}+\zeta_{5}\left(z_{3}, z_{5}, \widetilde{\Theta}, t\right) \\
& \dot{z}_{6}=-c_{6} z_{6}-z_{4}
\end{aligned}
$$

These are given the more compact form:

$$
\dot{Z}_{2}=A_{2} Z_{2}+\chi_{2}\left(Z_{2}, \widetilde{\Theta}, t\right)
$$

with:

$$
Z_{2}=\left[\begin{array}{llll}
z_{3} & z_{4} & z_{5} & z_{6}
\end{array}\right]^{T}, \quad \widetilde{\Theta}=\left[\begin{array}{lll}
\widetilde{J} & \widetilde{T}_{L} & \tilde{f}
\end{array}\right]^{T}
$$

$$
\begin{aligned}
& A_{2}=\left[\begin{array}{cccc}
-c_{3} & 0 & 1 / J & 0 \\
0 & -c_{4} & 0 & 1 \\
0 & 0 & -\left(c_{5}+f / J\right) & 0 \\
0 & -1 & 0 & -c_{6}
\end{array}\right], \\
& \chi_{2}\left(Z_{2}, \widetilde{\Theta}, t\right)=\left[\zeta_{3}\left(Z_{2} \widetilde{\Theta}, t\right) \quad 0 \quad \zeta_{5}\left(Z_{2}, \widetilde{\Theta}, t\right) \quad 0\right]^{T} \\
& \zeta_{3}\left(z_{3}, z_{5}, \widetilde{\Theta}, t\right) \stackrel{\text { def }}{=} \frac{\widetilde{J}}{J}\left(c_{3} z_{3}+\dot{\Omega}_{\text {ref }}\right)+\frac{\widetilde{T}_{L}}{J}+\frac{\tilde{f}}{J}\left(\Omega_{\text {ref }}-z_{3}\right) \\
& \zeta_{5}\left(z_{3}, z_{5}, \widetilde{\Theta}, t\right) \stackrel{\text { def }}{=} \frac{\widetilde{J}}{J}\left[\left(c_{3} \widehat{J}-\hat{f}\right)\left(c_{3} z_{3}+\dot{\Omega}_{r e f}\right)-c_{3} z_{5}\right] \\
& +\left(c_{3} \widehat{J}-\hat{f}\right) \frac{\widetilde{T}_{L}}{J}+\frac{\tilde{f}}{J}\left[\left(c_{3} \widehat{J}-\hat{f}\right) x_{3}+z_{5}\right]
\end{aligned}
$$

(2) Let the design parameters $c_{3}$ and $c_{5}$ be sufficiently large in the sense that $c_{3}>\frac{1}{2 j}$ and $c_{5}>\frac{1}{2 j}-\frac{f}{J}$. Then, all errors $\left(z_{3}, z_{4}, z_{5}, z_{6}, \widetilde{J}, \widetilde{T}_{L}, \tilde{f}\right)$ remain bounded and $\left(z_{3}, z_{4}, z_{5}, z_{6}\right)$ vanish asymptotically, whatever the initial conditions.

Proof. Part 1. Eq. (54a) and (54b) are immediately obtained from (35a) and (35b). Eq. (54c) is obtained substituting the control law (49) to $\left(u_{2}, u_{3}\right)$ on the right side of (39). Eq. (54d) is obtained substituting the control law (49) to $\left(\boldsymbol{u}_{2}, \boldsymbol{u}_{3}\right)$ on the right side of (44a). This proves Part 1.

Part 2. Substituting the control law (49) to $\left(u_{2}, u_{3}\right)$ on the right side of (48) yields:

$\dot{V}_{4}=-c_{3} z_{3}^{2}-c_{4} z_{4}^{2}-c_{5} z_{5}^{2}-c_{6} z_{6}^{2}-\frac{f}{J} z_{5}^{2}+\frac{1}{J} z_{3} z_{5}$

Using the inequality $\left|z_{3} z_{5}\right| \leqslant \frac{1}{2} z_{3}^{2}+\frac{1}{2} z_{5}^{2}$, one obtains from (57a):

$\dot{V}_{4} \leqslant-\left(c_{3}-\frac{1}{2 J}\right) z_{3}^{2}-c_{4} z_{4}^{2}-\left(c_{5}+\frac{f}{J}-\frac{1}{2 J}\right) z_{5}^{2}-c_{6} z_{6}^{2}$

As $c_{3}-\frac{1}{2 j}$ and $c_{5}+\frac{f}{J}-\frac{1}{2 j}$ are both positive, due to the assumption in Part 2, it follows from (57b) that $\dot{V}_{4}<0$ whenever the state vector $\left(z_{3}, z_{4}, z_{5}, z_{6}\right)$ is non-zero. Then, applying the Lasalle's invariant set principle, it follows that $V_{4}$ is bounded and $\left(z_{3}, z_{4}, z_{5}, z_{6}\right)$ converges to zero whatever the initial conditions.

Theorem 1 is established.

Remark 3. The derivatives $\dot{\Phi}_{r e f}$ and $\ddot{\Phi}_{\text {ref }}$ are obtained using the relation:

$\bar{\Phi}_{\text {ref }}=F\left(\bar{I}_{s}\right)=h_{0}+h_{1} \bar{I}_{s}+h_{2} \bar{I}_{s}^{2}+\cdots+h_{n} \bar{I}_{s}^{n}$

where

$\bar{I}_{s}=\sqrt{\left(x_{4}^{2}+x_{5}^{2}\right.}$

Specifically, one has:

$$
\begin{aligned}
\dot{\bar{\Phi}}_{\text {ref }}= & \frac{d F\left(\bar{I}_{s}\right)}{d t}=\frac{d F\left(\bar{I}_{s}\right)}{d \bar{I}_{s}} \frac{d \bar{I}_{s}}{d t}=\frac{d F\left(\bar{I}_{s}\right)}{d \bar{I}_{s}} \frac{x_{4} \dot{x}_{4}+x_{5} \dot{x}_{5}}{\bar{I}_{s}} \\
\ddot{\Phi}_{\text {ref }}= & \frac{d^{2} F\left(\bar{I}_{s}\right)}{d \bar{I}_{s}^{2}}\left(\frac{x_{4} \dot{x}_{4}+x_{5} \dot{x}_{5}}{\bar{I}_{s}}\right)^{2}+\frac{d F\left(\bar{I}_{s}\right)}{d \bar{I}_{s}} \\
& \times \frac{\left(\dot{x}_{4}\right)^{2}+x_{4} \ddot{x}_{4}+\left(\dot{x}_{5}\right)^{2}+x_{5} \ddot{x}_{5}}{\bar{I}_{s}}-\frac{d F\left(\bar{I}_{s}\right)}{d \bar{I}_{s}} \frac{\left(x_{4} \dot{x}_{4}+x_{5} \dot{x}_{5}\right)^{2}}{\bar{I}_{s}^{3}}
\end{aligned}
$$




\subsection{Achievement of PFC and DC link voltage regulation}

In this section, it will be formally shown that, for a specific class of reference signals, including periodic signals, the control objectives are achieved (in the mean) with an accuracy that depends, among others, on the network frequency $\omega_{e}$. The analysis is based on Eq. (19a) which, in view of (49) becomes:

$\dot{Z}_{1}=A_{1} Z_{1}+f\left(Z_{1}, t\right)+\rho\left(Z_{2}, t\right) g+h \dot{y}_{\text {ref }}^{*}$

with:

$$
\begin{aligned}
\rho\left(Z_{2}, t\right)= & \frac{-\left(\lambda_{J}\left(c_{3} z_{3}+\dot{\Omega}_{r e f}\right)+\lambda_{T_{L}}+\lambda_{f}\left(\Omega_{r e f}-z_{3}\right)\right)\left(z_{5}-\mu_{1}\right)}{a_{1} p^{2} \bar{\Phi}_{r}^{2}} \\
& -\frac{\left(c_{5}+c_{3}\right) z_{5}+\mu_{2}}{a_{1} p^{2} \bar{\Phi}_{r}^{2}}\left(z_{5}-\mu_{1}\right)-\frac{\left(-z_{4}-c_{6} z_{6}^{-} v_{2}\right)\left(v_{1}-z_{6}\right)}{4 a_{1}^{3} \bar{\Phi}_{r}^{2}}
\end{aligned}
$$

Theorem 2 (PFC and DC-link regulation). Consider the system including an $A C / D C$ power rectifier, a DC/AC inverter and an induction motor, connected as shown in Fig. 3. For control design purpose, the system is represented by its average model (6a)-(6e). Consider the controller defined by the control laws (10), (18) and (49), the parameter update laws (51)-(53) and the optimal flux generator (25). Let the reference signals $v_{d c r e f}, \Omega_{\text {ref }}$ and $\Phi_{\text {ref }}$ be selected such that $v_{\text {dcref }}>0, \Omega_{\text {ref }} \geqslant 0$ and $\Phi_{\text {ref }} \geqslant 0$ and $v_{\text {dcref }}$ and $\Omega_{\text {ref }}$, be periodic with period $N \pi / \omega_{e}$ (for some positive integer $N$ ), and suppose them to be time derivable ( $u$ to second order for $\Omega_{\text {ref }}$ ) with bounded derivatives. Finally, suppose the design parameters $\left(c_{1}, c_{2}, d\right)$ to be positive. Then, there exists a positive real $\varepsilon^{*}$ such that, if $0<\varepsilon<\varepsilon^{*}$, with $\varepsilon=1 / \omega_{e}$, then:

(1) The tracking error $z_{2}=y-y_{\text {ref }}$ and the tuning parameter $k$ are harmonic signals continuously depending on $\varepsilon$.

(2) Furthermore, one has:

(i) $\lim _{\varepsilon \rightarrow 0} z_{2}(t, \varepsilon)=0$ and $\quad($ ii $) \lim _{\varepsilon \rightarrow 0} k(t, \varepsilon)=\frac{1}{C} \frac{\bar{\rho}\left(0_{4}\right)}{b_{0}}$

where $\bar{\rho}\left(0_{4}\right)$ denotes the mean value of the periodic time function $\rho\left(0_{4}, t\right)$ and $0_{4} \in I R^{4}$ denotes the null vector

Proof. As $v_{\text {dcref }}$ and $\Omega_{\text {ref }}$ as well as their derivatives are constant or periodic (with period $N \pi / \omega_{e}$ ), it follows that the system (60) is periodically time-varying. Therefore, the averaging theory turns out to be a suitable framework to analyze its stability (see e.g. [6]). To this end, introduce the time-scale change $\tau=\omega_{e} t$ and the following signal changes:

$W_{1}(\tau)=Z_{1}(t), y_{\text {ref }}^{*}(t)=y_{\text {ref }}\left(\frac{N t}{2 \omega_{e}}\right), \quad \Omega_{\text {ref }}^{*}(t)=\Omega_{r e f}\left(\frac{N t}{2 \omega_{e}}\right)$

This readily implies that $y_{r e f}^{*}$ and $\Omega_{r e f}^{*}$ are in turn periodic, with period $2 \pi$, and:

$y_{\text {ref }}(t)=y_{\text {ref }}^{*}\left(\frac{2 \tau}{N}\right), \quad \Omega_{\text {ref }}(t)=\Omega_{\text {ref }}^{*}\left(\frac{2 \tau}{N}\right)$.

Also, it is easily seen that $\dot{W}_{1}(\tau)=d W_{1}(\tau) / d \tau=\varepsilon d Z_{1}(t) / d t=$ $\varepsilon \dot{Z}_{1}(t)$ with $\varepsilon=1 / \omega_{e}$. Then, using (63b) it follows from (60) that the state vector $W_{1}$ undergoes the following state equation:

$$
\begin{aligned}
\dot{W}_{1}= & \varepsilon A_{1} W_{1}+\varepsilon f_{1}\left(W_{1}, \tau, \varepsilon\right)+\varepsilon g \rho_{1}\left(W_{2}, \tau, \varepsilon\right)+h \dot{y}_{\text {ref }}^{*}\left(\frac{2 \tau}{N}\right) \\
= & \varepsilon A W_{1}+\varepsilon f_{1}\left(W_{1}, \tau, \varepsilon\right)+\varepsilon g \rho_{1}(0, \tau, \varepsilon)+h \dot{y}_{\text {ref }}^{*}\left(\frac{2 \tau}{N}\right) \\
& +\varepsilon g \tilde{\rho}_{1}\left(W_{2}, \tau, \varepsilon\right)
\end{aligned}
$$

with:

$$
\begin{array}{ll}
f_{1}\left(W_{1}, \tau, \varepsilon\right)=f\left(W_{1}, \varepsilon \tau\right), & W_{2}(\tau)=Z_{2}(t) \\
\rho_{1}\left(W_{2}, \tau, \varepsilon\right)=\rho\left(W_{2}, \varepsilon \tau\right) & \tilde{\rho}_{1}\left(W_{2}, \tau, \varepsilon\right)=\rho_{1}\left(W_{2}, \tau, \varepsilon\right) \\
\quad-\rho_{1}(0, \tau, \varepsilon) &
\end{array}
$$

It readily follows from $(64 b)$ that:

$f_{1}\left(W_{1}, \tau, \varepsilon\right)=\left[\begin{array}{lll}0 & \left(b_{0} w \cos (2 \tau)+b_{2} w_{1} \cos (\tau)\right) & 0\end{array}\right]^{T}$

where the following notations are adopted in coherence with (19b):

$W_{1}=\left[\begin{array}{lll}w_{1} & w_{2} & w\end{array}\right]^{T}$

By Theorem 1 and (64c) one has $W_{2}(\tau) \rightarrow 0$ as $\tau \rightarrow \infty$. Since $\rho_{1}\left(W_{2}, \tau, \varepsilon\right)$ is continuous in $W_{2}$, it follows that $\tilde{\rho}_{1}\left(W_{2}, \tau, \varepsilon\right) \rightarrow 0$ as $\tau \rightarrow \infty$. Then, according to the averaging theory, one gets stability results regarding the system of interest (64a) and (64b) by analyzing the averaged system defined by:

$\dot{W}_{1}=\varepsilon A \bar{W}_{1}+\varepsilon \bar{f}_{1}\left(\bar{W}_{1}\right)+\varepsilon g \bar{\rho}_{1}\left(\mathbf{0}_{4}\right)$

where

$\bar{f}_{1}(\bar{W}) \stackrel{\text { def }}{=} \lim _{\varepsilon \rightarrow 0} \frac{1}{2 \pi \mathrm{N}} \int_{0}^{2 \pi N} f_{1}(\bar{W}, \tau, \varepsilon) d \tau$

$\bar{\rho}_{1}\left(\bar{W}_{2}\right) \stackrel{\text { def }}{=} \lim _{\varepsilon \rightarrow 0} \frac{1}{2 \pi \mathrm{N}} \int_{0}^{2 \pi N} \rho_{1}(0, \tau, \varepsilon) d \tau$

Note that the fourth term on the right side of (64a) has not been accounted for in (66a) because its average value is null, due to the periodicity (with period $2 \pi$ ) of $y_{\text {ref. }}^{*}$. From (64c), one has:

$\bar{f}_{1}(\bar{W})=\left[\begin{array}{lll}0 & 0 & 0\end{array}\right]^{T}$

In view of (67) the average system (66a) simplifies to:

$\dot{\bar{W}}_{1}=\varepsilon A_{1} \bar{W}_{1}+\varepsilon g \bar{\rho}_{1}\left(\mathbf{0}_{4}\right)$

Now, let us check that $A_{1}$ is in turn Hurwitz. Its characteristic polynomial is:

$\operatorname{det}\left(\lambda I-A_{1}\right)=\lambda^{3}+\left(c_{1}+d\right) \lambda^{2}+d\left(c_{1}+c_{2}\right) \lambda+d c_{1} c_{2}$

Applying for instance the well known Routh's algebraic criteria, it follows that all zeros of the polynomial (69) have negative real parts if the coefficients $c_{1}, c_{2}$ and $d$ are positive which actually is presently the case. Hence, the matrix $A_{1}$ is Hurwitz implying that the autonomous part of the linear system (68) is globally exponentially stable. Then, the solution of the system (68) satisfies:

$\lim _{t \rightarrow \infty} \bar{W}_{1}(t)=-\bar{\rho}_{1}\left(0_{4}\right) A_{1}^{-1} g, \quad$ exponentially and whatever $\bar{W}_{1}(0)$

The exponential feature of the convergence is due to the linearity of (68). From (70), it follows that the state vector

$W_{1}^{*} \stackrel{\text { def }}{=}-\bar{\rho}_{1}\left(0_{4}\right) A_{1}^{-1} \quad g \in I R^{3}$

is a globally exponentially stable equilibrium of the average system (68). Now, invoking averaging theory, e.g. Theorem 10.4 in [6], we conclude that there exists a positive real constants $\varepsilon^{*}$ such that, for all $0<\varepsilon<\varepsilon^{*}$, the differential equation (64a) has a $2 \pi$-periodic solution $W_{1}(\tau)=W_{1}(\tau, \varepsilon)$, that continuously depends on $\varepsilon$ and that:

$\lim _{\varepsilon \rightarrow 0} W_{1}(\tau, \varepsilon)=W_{1}^{*}, \quad$ exponentially

The same result applies to the differential equation (60) using the relation $Z_{1}(t)=W_{1}(\tau)$ with $\tau=\omega_{e} t$. That is, $Z_{1}(t)=Z_{1}(t, \varepsilon)$ is $\left(2 \pi / \omega_{e}\right)$-periodic, continuously depends on $\varepsilon$ and satisfies:

$\lim _{\varepsilon \rightarrow 0} Z_{1}(t, \varepsilon)=W_{1}^{*}$ 
This establishes Part 1 of Theorem 2.

To prove Part 2, note that from (71) one has:

$W_{1}^{*}=-\bar{\rho}_{1}\left(0_{4}\right) A_{1}^{-1} g$

Also, it is readily checked using (19d) and (19e) that:

$W_{1}^{*}=-\bar{\rho}_{1}\left(0_{4}\right) A_{1}^{-1} g=-\bar{\rho}_{1}\left(0_{4}\right)\left[\begin{array}{lll}0 & \frac{d-C b_{0} b_{3}}{C b_{0} b_{1}} & \frac{-1}{C b_{0}}\end{array}\right]^{T}$

Furthermore, it is obviously seen from (19b) that:

$$
\frac{d-C b_{0} b_{3}}{C b_{0} a_{1}}=0
$$

Consequently, one gets from (72b), (73b) and (73c) that:

$$
\begin{aligned}
& \lim _{\varepsilon \rightarrow 0} z_{2}(t, \varepsilon)=w_{2}^{*}=0 \\
& \lim _{\varepsilon \rightarrow 0} k(t, \varepsilon)=w^{*}=\frac{\bar{\rho}_{1}\left(0_{4}\right)}{C b_{0}}
\end{aligned}
$$

Finally, notice that:

$$
\begin{aligned}
\bar{\rho}_{1}\left(0_{4}\right) & =\lim _{\varepsilon \rightarrow 0} \frac{1}{2 \pi N} \int_{0}^{2 \pi N} \bar{\rho}_{1}\left(0_{4}, \tau, \varepsilon\right) d \tau(\text { using (66c)) } \\
& =\lim _{\varepsilon \rightarrow 0} \frac{1}{2 \pi N} \int_{0}^{2 \pi N} \rho\left(0_{4}, \varepsilon \tau\right) d \tau(\text { using (64b)) }
\end{aligned}
$$

Introducing the variable change $\tau=\omega_{e} t$, (75a) becomes:

$$
\bar{\rho}_{1}\left(0_{4}\right)=\lim _{\varepsilon \rightarrow 0} \frac{\omega_{e}}{2 \pi N} \int_{0}^{2 \pi N / \omega_{e}} \rho\left(0_{4}, t\right) d t=\bar{\rho}\left(0_{4}\right)
$$

This, together with (74a) and (74b), establishes Part 2-b and completes the proof of Theorem 2 .

\section{Remark 4}

(a) The motor speed and the rotor flux norm both converge to their respective references because the errors $\left(z_{3}, z_{5}\right)$ converge to zero, a result of Theorem 1 .

(b) Using Proposition 1 and the fact that the tuning parameter $k$ and its time derivative are available, we gets that the error $z_{1}=x_{1}-x_{1}^{*}=i_{e}-k v_{e}$ converges exponentially fast to zero. The importance of Theorem 2 (part 2) lies (partly) in the fact that the (time varying) parameter $k$ does converge to a fixed value (up to a harmonic error that depends on $\omega_{e}$ ). This demonstrates that the PFC requirement is actually fulfilled with an accuracy that depends on $\omega_{e}$. The larger $\omega_{e}$ is, the more accurate the PFC quality. It will be seen in the next simulation study that the usual value $\omega_{e}=100 \pi \mathrm{rd} / \mathrm{s}$ leads to a quite acceptable quality.

(c) Theorem 2 (Part 2) also demonstrates that the tracking objective is achieved (in the mean) for the DC-link squared voltage $y=x_{2}^{2}=v_{d c}^{2}$ with an accuracy that depends on the voltage network frequency $\omega_{e}$. The class of admissible references $v_{\text {dcref }}$ and $\Omega_{\text {ref }}$ includes periodic signals with period $N \pi / \omega_{e}$. That is, these signals must oscillate less rapidly than the network voltage.

(d) The fact that the tracking error $z_{2}=v_{d c}^{2}-v_{d c r e f}^{2}$ is harmonic proves the existence of output ripples. Theorem 2 (Part 2) ensures that the effect of these ripples is insignificant if $\omega_{e}$ is sufficiently large. It will be observed through simulations that the value $\omega_{e}=100 \pi \mathrm{rd} / \mathrm{s}$ leads to sufficiently small ripples.

\section{Simulation}

The experimental setup has been simulated within the Matlab/ Simulink environment. The simulated system has the following characteristics:
- Supply network: $v_{e}(t)=\sqrt{2} \cdot E \cos \left(\omega_{e} t\right)$ a single phase $220 \mathrm{~V} \backslash 50 \mathrm{~Hz}$.

- AC/DC/AC converters: $L_{1}=15 \mathrm{mH} ; C=1.5 \mathrm{mF}$; modulation frequency: $10 \mathrm{KHz}$.

The induction machine characteristics are summarized in Table 1.

The experimental protocol is described by Figs. 9-11. Accordingly, the inertia moment $J$ and viscous friction coefficient $f$ deviate from their nominal values on some time intervals (Figs. 9 and 10). The applied load torque $T_{L}$ (Fig. 11) and rotor speed reference $\Omega_{\text {ref }}$ (Fig. 18) are chosen so that the induction machine works in two zones of its magnetic characteristic and operates both as motor and generator. Indeed, the induction machine operates in motor mode in the interval $[0,16 s]$ and in generator mode in the interval $[16 s, 20 s]$. The DC-link voltage reference is constant, i.e. $v_{\text {dcref }}=600 \mathrm{~V}$.

Recall that the nonlinear adaptive controller to be illustrated is described by: (i) the control laws (10), (18) and (49); (ii) the parameter update laws (51)-(53); and (iii) the optimal flux reference generator (25). The following values of the controller design parameters proved to be suitable:

$c_{1}=1000, \quad c_{2}=30, \quad c_{3}=100, \quad c_{4}=400, \quad c_{5}=500$,

$c_{6}=1000, d=100$

The above adaptive controller will be compared with its simplified (constant flux reference) version obtained keeping the flux reference constant equal to its nominal value, i.e. $0.56 \mathrm{~Wb}$. To avoid confusion, the two controllers will be referred to Optimal Flux Reference (OFR) adaptive controller and Constant Flux Reference (CFR) adaptive controller.

The performances of both controllers are illustrated by Figs. 12-20. It is seen from Fig. 12 that the input current $i_{e}$ is higher with the CFR adaptive controller than with the OFR. Fig. 13 shows the reference and measured input current $i_{e}$ response of the OFR controller: it is observed that the current amplitude changes whenever the speed reference or the load torque vary. However, the current frequency is insensitive to these changes. Specifically, the current remains most time either in phase (motor mode) or opposed phase (generator mode) with the supply net voltage, complying with the PFC requirement. This is further demonstrated by Figs. 14-16 which show that the ratio $k$ takes a constant value after short transient periods

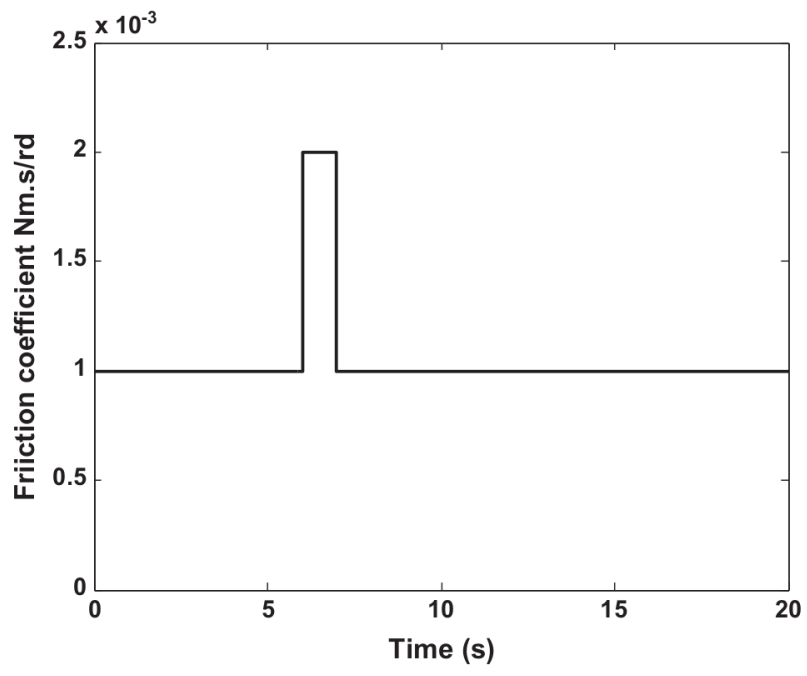

Fig. 9. Friction coefficient variation. 


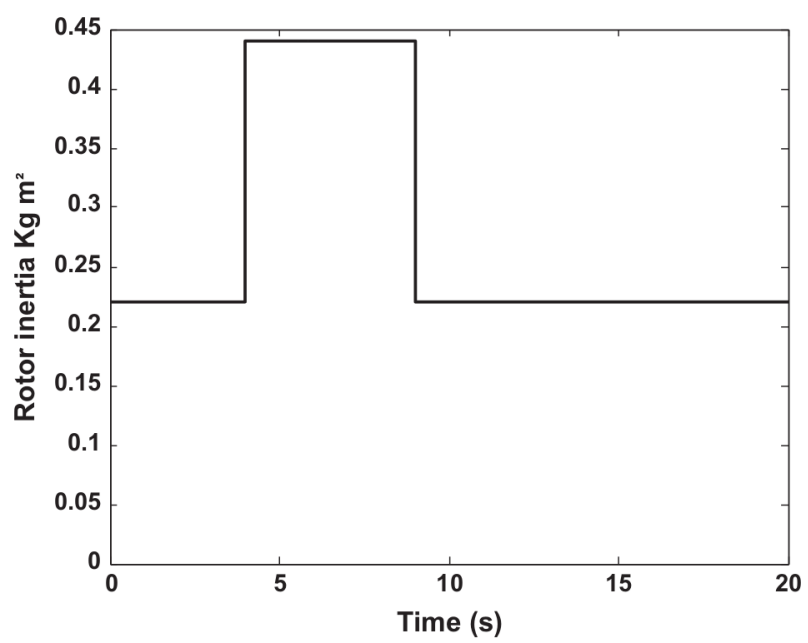

Fig. 10. Rotor inertia variation.

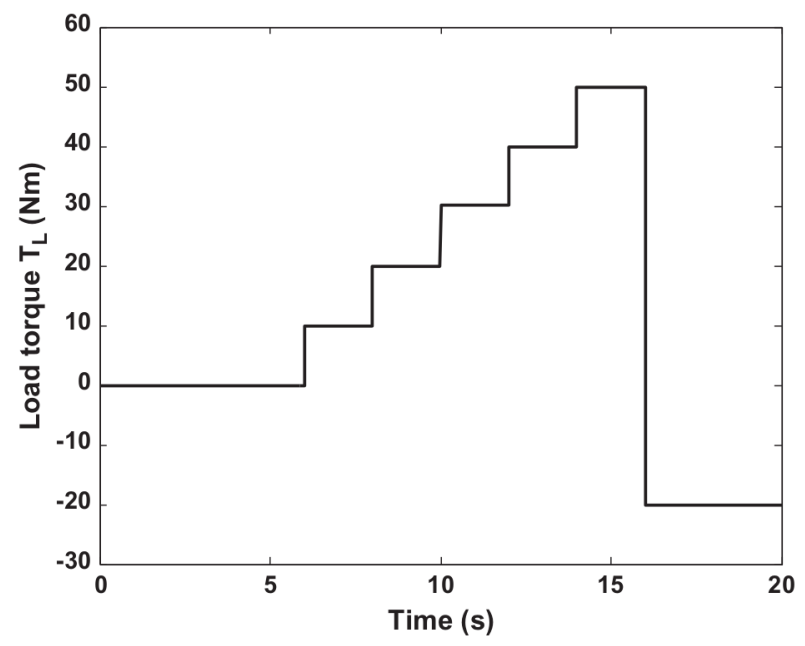

Fig. 11. Applied load torque.

following changes in the references signal and load torque. Note that the ratio $k$ is negative when the network gets back energy. Fig. 17 shows that the DC-link voltage $x_{2}=v_{d c}$ is well regulated and quickly settles down after each change in the speed reference or the load torque. As stipulated by Theorem 2, and commented on in Remark $4 \mathrm{c}$, the DC link voltage $v_{d c}$ is subject to small amplitude ripples oscillating at the supply net frequency $\omega_{e}$. Fig. 18 shows that both controllers ensure a perfect asymptotic speed reference tracking despite the uncertainty and changes of the mechanical parameters (load torque $T_{L}$, rotor inertia $J$ and viscous friction coefficient $f$ ). Fig. 19 shows the resulting (state-dependent) optimal flux reference (for the OFR adaptive controller) and the constant flux reference (for the simplified CFR adaptive controller). It is clearly seen that the statedependent flux reference varies significantly in function of the load torque and the speed. Fig. 20 shows the absorbed stator currents for both controllers, in various operation conditions. It is seen that the OFR controller requires a smaller current than the constant flux controller. This difference is more significant in presence of low load torques, because in this situation the optimal flux reference is most different from the constant flux reference.

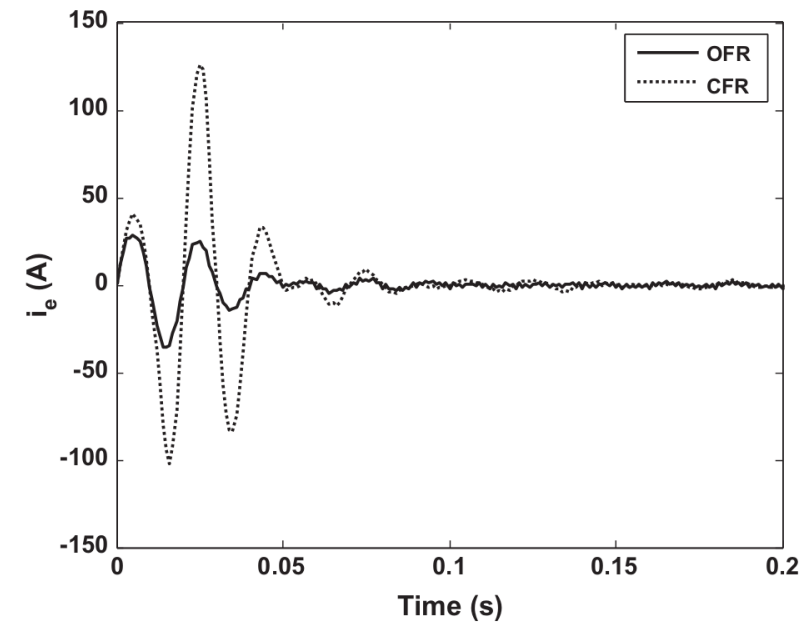

Fig. 12. Zoom on the input current $i_{e}$ response (A) over the interval $\left[\begin{array}{ll}0 & 0.2 s\end{array}\right]$ (solid: OFR controller, dotted: CFR controller).

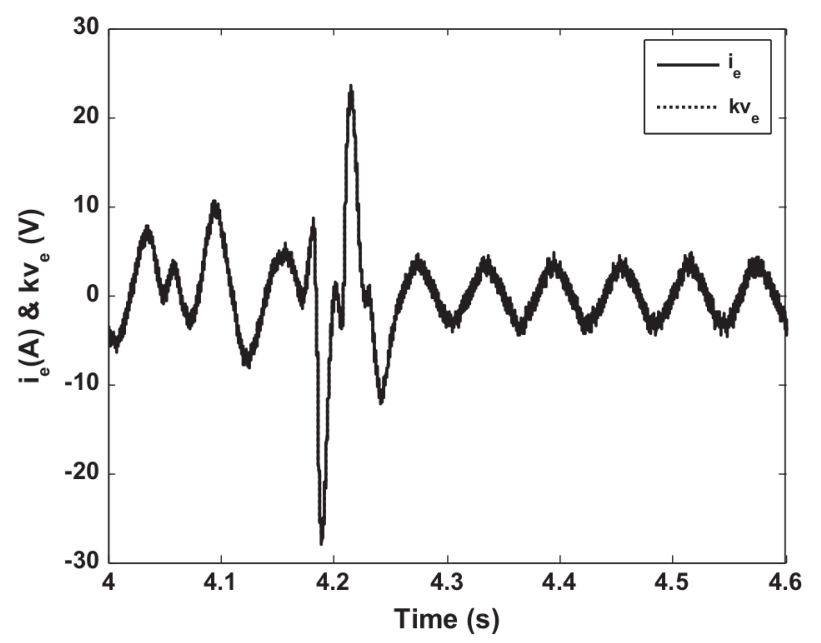

Fig. 13. Zoom on the reference and measured input current $i_{e}(\mathrm{~A})$ over the interval $\left[\begin{array}{ll}4 & 4.6 s]\end{array}\right]$ when using the OFR controller.

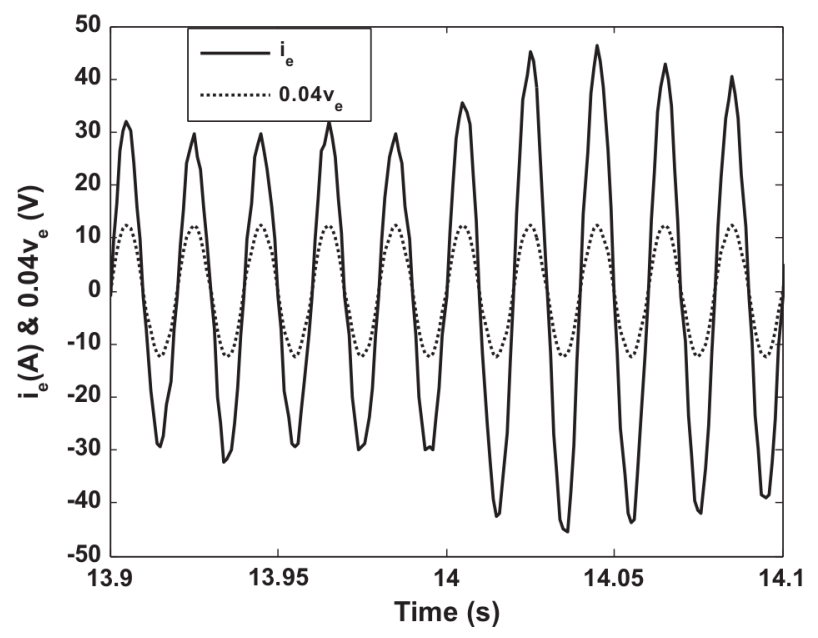

Fig. 14. Unitary power factor checking with the OFR controller when the induction machine absorbs energy (motor mode operation). 


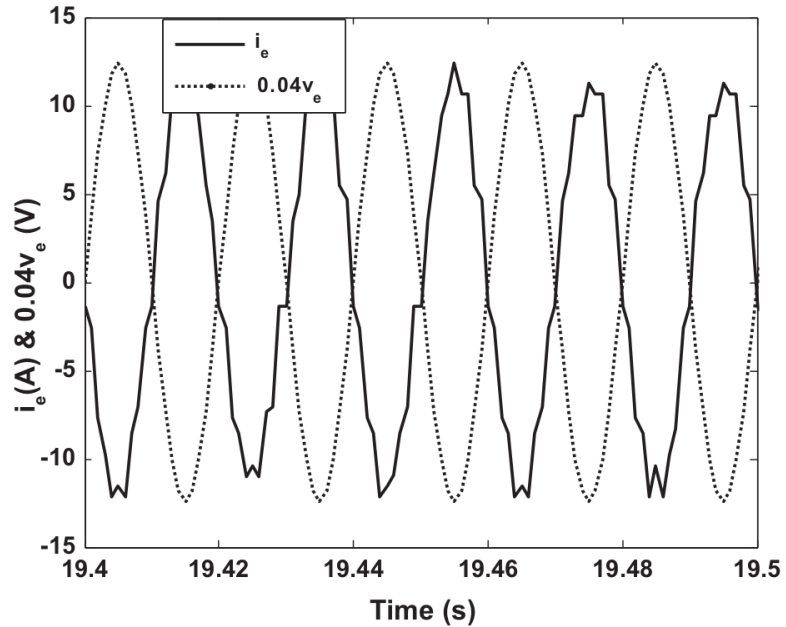

Fig. 15. Unitary power factor checking with the OFR controller when the machine restores energy (generator mode operation).

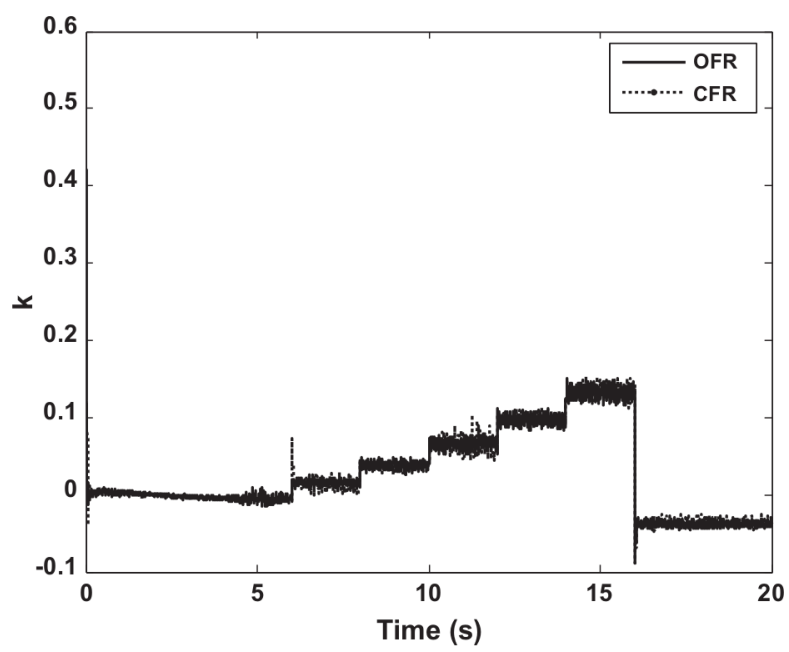

Fig. 16. Tuning parameter $k$. Solid: OFR controller; dotted: CFR controller.
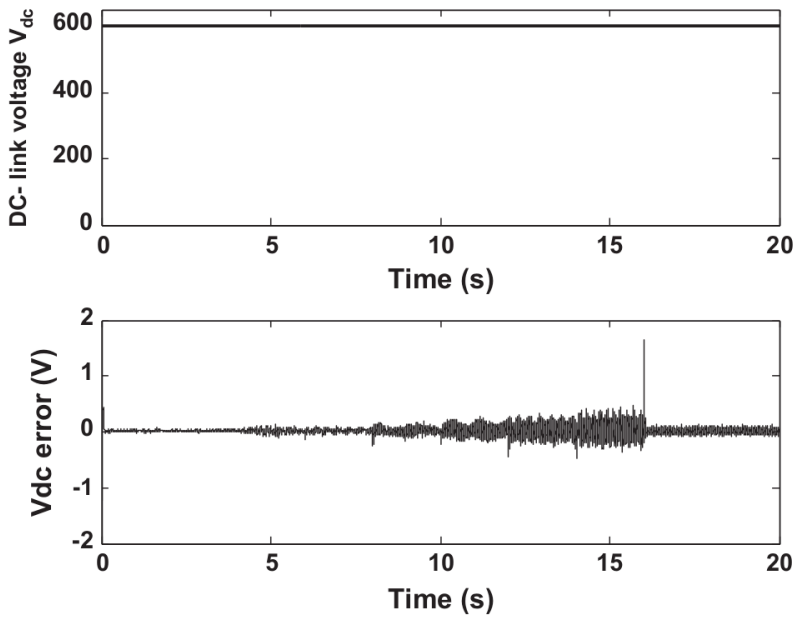

Fig. 17. DC-link voltage $v_{d c}(\mathrm{~V})$ response. Upper: reference and measured DC-link voltage; lower: DC-link voltage error control.

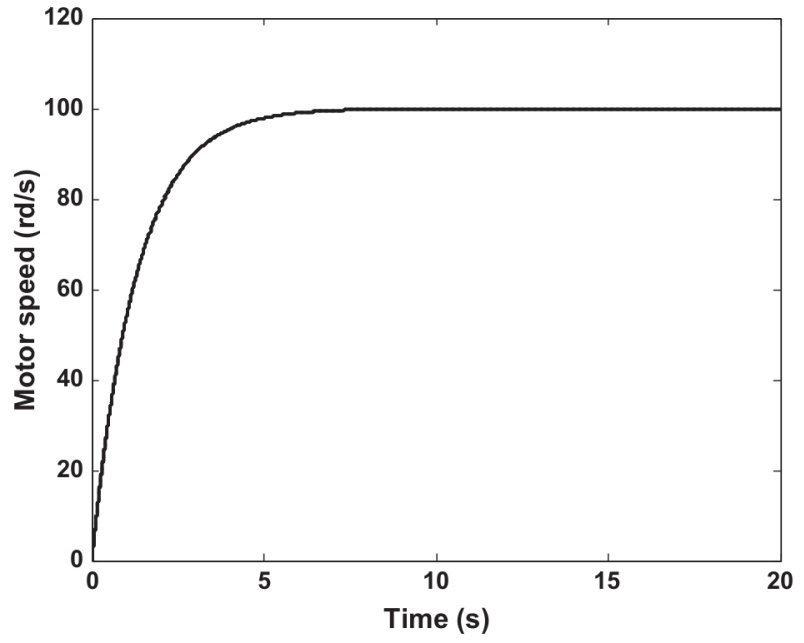

Fig. 18. Rotor speed $\Omega(\mathrm{rd} / \mathrm{s})$ response: the identical speed responses obtained by the OFR controller and the constant flux controller.

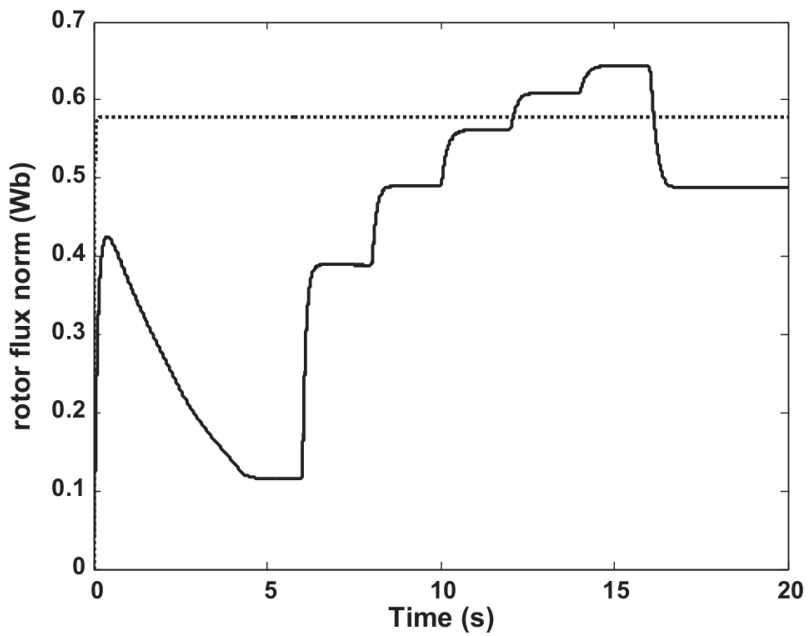

Fig. 19. Rotor flux norm reference $(\mathrm{Wb})$ (solid: state-dependent optimized flux, dotted: constant flux).
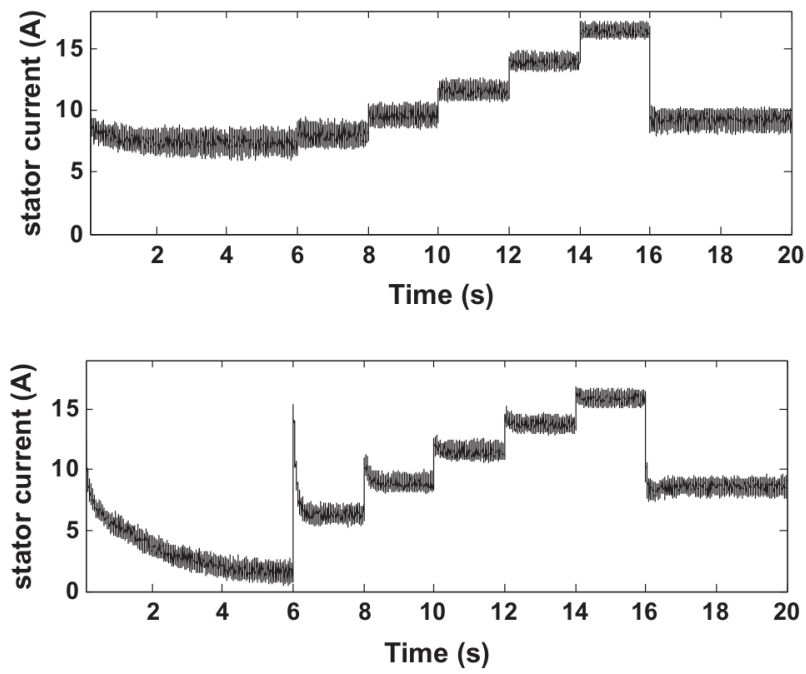

Fig. 20. Absorbed stator current (A) (Upper: constant flux controller. Lower: OFR controller). 


\section{Conclusion}

The problem of controlling associations including an AC/DC rectifier, a DC/AC inverter and induction motor has been addressed with consideration of magnetic characteristic saturation. The system dynamics have been described by the averaged 7th order nonlinear state-space model (6a)-(6g). Based on such a model, a multiloop nonlinear adaptive controller has been designed and analyzed in several steps. The controller consists of: (i) the control laws (10), (18) and (49); (ii) the parameter update laws (51)-(53); and (iii) the optimal flux reference generator (25). Their performances have been analyzed using tools from the Lyapunov stability and averaging theory. It has been formally established that the controller actually meets the objectives it has been designed to, i.e. (i) almost unitary power factor; (ii) well regulated DC-link voltage $\left(\mathrm{v}_{d c}\right)$; (iii) satisfactory rotor speed reference tracking over a wide range of mechanicals parameters variation; and (iv) tight optimization and regulation rotor flux norm for minimizing the stator current absorbed. These results have been confirmed by a simulation study. To the author's knowledge, it is the first time that a so complete formal design and analysis framework has been developed for inductions motors in presence of magnetic saturation control design.

\section{References}

[1] Abdelaziz M, Ghedjati K. Control by feedback linearization of the torque and the flux of the induction motor. In: 7th WSEAS international conference on systems theory and scientific computation, Athens, Greece, vol. 7; 2007. p. 8490.

[2] De Wit Canudas C, Ramierz J. Optimization, discretization, observers and control of inductions motors. Paris: Hermes Science Europe Ltd.; 2000.

[3] Mahmuda MA, Potaa HR, Hossainb MJ. Full-order nonlinear observer-based excitation controller design for interconnected power systems via exact linearization approach. Int J Electr Power Energy Syst 2012;41:54-62.

[4] Jasinski M, Cichowlas M, Kazmierkowski MP. Direct control for AC/DC/AC converter-fed induction motor with active filtering function. Int J Comput Math Electrical Electronic Eng 2006;25:235-42.
[5] Kazmierkowski MP. Control strategies for PWM rectifier/inverter-fed induction motors. In: IEEE international symposium on industrial electronics (ISIE 2000), vol. 1; 2000. p. 15-21.

[6] Khalil H. Nonlinear systems. NJ, USA: Prentice Hall; 2003.

[7] Krstic M, Kanellakopoulos I, Kokotovic P. Nonlinear and adaptive control design. John Willey \& Sons, Inc.; 1995.

[8] Leonard W. Control of electrical drives. NY: Springer; 2001.

[9] Zaveri T, Bhalja BR, Zaveri Naimish. A novel approach of reference current generation for power quality improvement in three-phase, three-wire distribution system using DSTATCOM. Int J Electrical Power Energy Syst 2011;33(10):1702-10.

[10] Michael J, Ryan D, Rik W. Modeling of sinewave inverters: a geometric approach. In: IEEE industrial electronics conference (IECON 1998), vol. 1; 1998. p. 396-401.

[11] Montanan M, Peresada S, Tilli A. A speed-sensorless indirect field-oriented control for induction motors based on high gain speed estimation. Automatica 2006;42(10):1637-50.

[12] Moreno-Eguilaz JM, Peracaula J. Efficiency optimization for induction motor drives: past, present and future. In: Electrimacs 99: modelling and simulation of electric machines converters and systems. IMACS No. 6, Lisboa, Potugal; 1999 p. $187-91$.

[13] Ouadi H, Giri F, De Leon-Morales J, Dugard L. Accounting for magnetic characteristic nonlinearity in designing induction motor observer. In: IFAC symposium on nonlinear control systems, NOLCOS, Stuttgart, Germany; 2004. p. $1409-14$.

[14] Ouadi H, Giri F. Induction motor robust adaptive control. In: Mediterranean conference on control and automation - MED 2002, Lisbon Portugal; 2002.

[15] Novotnak RT, Chiasson J, Bodson M. High performance motion control of an induction motor with magnetic saturation. IEEE Trans Contr Syst Technol 1999;7:315-27.

[16] Singh B, Bhuvaneswari G, Garg V. Improved power quality AC-DC converter for electric multiple units in electric traction. In: Power India conference; 2006. p. 6

[17] Sira H, Silva R. Control design techniques in power electronics devices. Springer; 2006.

[18] Hansen S, Malinowski M, Blaabjerg F, Kazmierkowski MP. Sensorless control strategies for PWM rectifier. In: Applied power electronics conference and exposition, 2000. APEC 2000, vol. 2; 2000. p. 832-8 [Fifteenth annual IEEE].

[19] Traoré D, Plestan F, Glumineau A, de Leon J. Sensorless induction motor: high order sliding mode controller and adaptive interconnected observer. IEEE Trans Ind Electron 2008:55:3818-27.

[20] Saha S, Aldeen M, Tana CP. Fault detection in transmission networks of power systems. Int J Electrical Power Energy Syst 2011;33(4):887-900.

[21] Singh B, Jayaprakash P, Kothari DP. New control approach for capacitor supported DSTATCOM in three-phase four wire distribution system under non-ideal supply voltage conditions based on synchronous reference frame theory. Int J Electrical Power Energy Syst 2011;33(5):1109-17.

[22] Ouadi H, Giri F, Dugard L. Accounting for magnetic saturation in induction machines modeling. Int J Model Identif Control 2011;14:27-36. 